\title{
1 Genetic, parental and lifestyle factors influence telomere length
}

2 Sergio Andreu-Sánchez ${ }^{1,2 *}$, Geraldine Aubert ${ }^{3,4 *}$, Aida Ripoll-Cladellas ${ }^{5 *}$, Sandra Henkelman ${ }^{6 *}$,

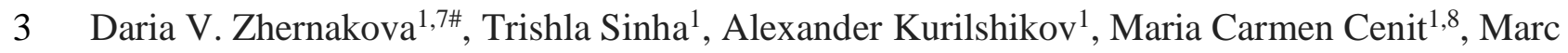

4 Jan Bonder ${ }^{1,9,10}$, Lifelines cohort study, Lude Franke ${ }^{1}$, Cisca Wijmenga ${ }^{1}$, Jingyuan Fu ${ }^{1,2}$,

5 Monique G.P. van der Wijst ${ }^{1}$, Marta Melé 5 , Peter Lansdorp ${ }^{3,6,11 \$}$, Alexandra Zhernakova ${ }^{1 \$}$

$7{ }^{1}$ Department of Genetics, University of Groningen, University Medical Center Groningen,

8 Groningen, the Netherlands

$9{ }^{2}$ Department of Pediatrics, University of Groningen, University Medical Center Groningen,

10 Groningen, the Netherlands

$11{ }^{3}$ Terry Fox Laboratory, British Columbia Cancer Research Center, Vancouver, BC, Canada

$12 \quad{ }^{4}$ Repeat Diagnostics Inc., Vancouver, BC, Canada

13 5ife Sciences Department, Barcelona Supercomputing Center, 08034, Barcelona, Catalonia,

14 Spain

$15{ }^{6}$ European Research Institute for the Biology of Ageing, University of Groningen, Groningen,

16 the Netherlands

$17{ }^{7}$ Laboratory of Genomic Diversity, Center for Computer Technologies, ITMO University, St.

18 Petersburg, 197101, Russia

$19{ }^{8}$ Microbial Ecology, Nutrition, and Health Research Unit. Institute of Agrochemistry and Food

20 Technology (IATA-CSIC), 46980 Paterna-Valencia, Spain

$21{ }^{9}$ Division of Computational Genomics and Systems Genetics, German Cancer Research Center

22 (DKFZ), 69120 Heidelberg, Germany

$23{ }^{10}$ European Molecular Biology Laboratory, Genome Biology Unit, 69117 Heidelberg, Germany 
$24{ }^{11}$ Departments of Hematology and Medical Genetics, University of British Columbia,

\section{Vancouver, BC, Canada}

$26 *$ Shared first author

$27 \quad$ \# Second author

28 Shared last authors 


\section{Abstract}

The average length of telomere repeats (TL) declines with age and is considered to be a marker of biological ageing. Here, we measured TL in six blood cell types from 1,046 individuals using the clinically validated Flow-FISH method. We identified remarkable cell-type-specific variations in TL. Host genetics, environmental, parental and intrinsic factors such as sex, parental age, and smoking are associated to

34 variations in TL. By analysing the genome-wide methylation patterns, we identified that the association of maternal, but not paternal, age to TL is mediated by epigenetics. Coupling these measurements to single-cell RNA-sequencing data for 62 participants revealed differential gene expression in T-cells. Genes negatively associated with TL were enriched for pathways related to translation and nonsense-mediated decay. Altogether, this study addresses cell-type-specific differences in telomere biology and its relation to cell-typespecific gene expression and highlights how perinatal factors play a role in determining TL, on top of genetics and lifestyle.

\section{Introduction}

43 With an increasingly ageing worldwide population, age-related diseases pose a great burden in clinical care and

44 socioeconomics. Healthy ageing is the goal to counter this trend, but this term is complex and not easily defined.

45 Several markers for premature or delayed ageing have been suggested, including telomere length ${ }^{1,2}$, DNA

46 methylation ${ }^{3,4}$ and thymic function ${ }^{5}$. Telomeres are repetitive DNA structures located at the chromosome ends and,

47 together with their associated proteins, play a fundamental role in chromosomal stability ${ }^{6,7}$. Telomeres are known to

48 decrease with age $^{2,8}$ as a result of multiple factors, including limiting telomerase activity ${ }^{9}$, the end-replication

49 problem ${ }^{1}$, end-processing ${ }^{10}$ and oxidative stress ${ }^{11}$. Both genetic and environmental factors are known to influence

50 telomere length. Several genetic loci have been associated with telomere length ${ }^{12-16}$, with heritability estimates

51 ranging from $34 \%{ }^{17}$ to $82 \%{ }^{18,19}$. However, heritability estimates often cannot distinguished between true genetic

52 determinants and early life factors such as parental or environmental exposures that could affect telomere length

53 during adulthood ${ }^{18}$. This is especially true in twin-based studies, where the early life exposures are confounded with 54 genetic effects.

55 Importantly, most telomere length analyses carried out to date focused on blood leukocytes and did not explore cell-

56 type- and tissue-specific variability. A recent study examining the variability of telomere length in a wide range of 
57 post-mortem tissues ${ }^{20}$ showed that, although whole blood telomere measurements might be a proxy for and

58 synchronous with those of other tissues ${ }^{21}$, there are significant tissue-specific differences. However, this post-

59 mortem tissue study did not address the possibility of telomere length differences between different cell types within

60 a tissue. In addition, most large studies to date have used PCR or Luminex-based methods to measure relative

61 telomere length in isolated genomic DNA, and these approaches have shown reproducibility biases ${ }^{22,23}$ that could

62 potentially explain the heterogeneous and contradicting findings ${ }^{24}$.

63 Telomere length may have important physiological consequences ${ }^{25}$. It has been proposed that telomere length might

64 regulate gene expression ${ }^{26-29}$ but also that gene expression can directly contribute to telomere length attrition or 65 conservation ${ }^{30,31}$. Given the observed variability in telomere length with cell population ${ }^{20}$, it is conceivable that this

66 will be related with cell-type-specific expression patterns, which have not been investigated to date.

67 Here, we explore telomere variation using Flow-FISH telomere length measurements in Lifelines Deep (LLD), a

68 well-characterised population cohort from the Netherlands ${ }^{32}$. We measured telomere length in six different cell types

69 in 1,046 participants. By combining this data with genetic information available for LLD participants and with rich

70 phenotypic information that includes blood cell counts and immune markers, self-reported diseases, birth-related

71 phenotypes, parental diseases and behaviour, epigenomics profiles and single-cell expression patterns in 62

72 individuals, we determined the major contributors to telomere length variation. Specifically, we studied: (1) the

73 difference in telomere length across the six blood cell types, (2) the relationship between leukocyte telomere length

74 with other ageing markers, (3) the correlation of telomere ageing markers with biochemical, parental and clinical

75 phenotypes and mortality, (4) the contribution of genetic and non-genetic factors to variations in telomere length

76 variation and (5) the cell-type-specific changes in gene expression associated with cell-type-specific telomere length

77 variation, which may pinpoint major functional pathways related to telomere variability.

79 Results

\section{Telomere length captures biological variability other than age}

$81 \mathrm{LLD}^{32}$ is a population cohort from the northern Netherlands that includes participants with a wide age range (mean

8243.9 years $\pm 13.7 \mathrm{sd}$, $\min 18.0, \max 81.4$ ) for whom we have deep phenotypic and molecular information [available

83 data illustrated in Supplementary Figure 1, for descriptive statistics see Supplementary Table 1]. In 1,046 LLD

84 participants, Flow-FISH ${ }^{33}$ was used to measure the telomere length of six blood cell types: granulocytes, 
85 lymphocytes, B-cells (CD45RA+CD20+), naïve T-cells (CD45RA+ CD20-), memory T-cells (CD45RA-) and NK86 cells/fully differentiated T-cells (CD45RA+CD57+) (hereafter referred to as NK-cells ) [Figure 1A]. We found that 87 all six cell-type-specific telomere lengths decreased with age [Figure 1B] and were, on average, shorter in males 88 than females over the entire age range [Figure 1C][Supplementary Figure 2B for all cell types]. We also observed 89 a similar moderate negative correlation between age and telomere length among cell types (Pearson correlation, 90 maximum $r=-0.43$, minimum $r=-0.33$ ) [Figure 1D]. These findings agree with those of a Flow-FISH-based study 91 in a North American cohort ${ }^{9}$, although the two cohorts differed in their participant recruitment selection criteria.

92 Nevertheless, in the overlapping age ranges, both studies find comparable telomere lengths [Supplementary Figure

93 2A], which supports the accuracy of our measurements. We observe that naïve T-cells and B-cells have the longest

94 telomeres on average, whereas NK- and memory T-cells show significantly shorter telomeres than other cell types

95 (Paired Wilcoxon-test, $\mathrm{p}<2 \times 10^{-16}$. While naïve T-cells showed the highest mean telomere length [Figure 1A], they

96 also showed the largest negative association of telomere length and age (linear model, slope of -0.034), from an

97 average $8.59 \mathrm{~Kb}$ in the $<32.9$ years age group (first quantile) to an average $7.32 \mathrm{~Kb}$ in individuals $>52.7$ years

98 (fourth quantile) [Figure 1B]. The rate of telomere loss we observe in naïve T-cells matches previous observations ${ }^{9}$

99 and does not support production of naïve T-cells from more primitive precursors after puberty and thymus

100 involution $^{34}$. Instead, we assume that naïve T-cells are maintained after puberty by homeostatic mechanisms that are

101 likely to involve cell divisions that result in telomere loss, but these processes are currently poorly understood.

102 Next, we compared telomere length with other biological age markers, including the methylation-based Hannum ${ }^{35}$

103 age-index and signal joint T-cell receptor excision circles (sjTRECs) expression (CT values of a qPCR), which

104 represents thymic TCR maturation for a given individual ${ }^{36}[$ Figure 1D]. Here, we observed that both methylation

105 age and sjTREC were more strongly associated with chronological age than telomere length, and neither was highly

106 correlated with telomere length [Figure 1D]. After removing the variability attributed to chronological age,

107 methylation age and sjTREC were negatively associated (Pearson correlation, $\mathrm{r}=-0.36$ ), but we found no association

108 to telomere length and telomere lengths remained highly correlated between different cell types [Supplementary

\section{Figure 2D].}

110 Overall, these findings suggest that telomere length captures biological variation other than chronological age, and 111 the source of this variation is distinct compared to other ageing markers measured, specifically methylation age and 112 thymic function. 
bioRxiv preprint doi: https://doi.org/10.1101/2021.12.14.472541; this version posted December 15, 2021. The copyright holder for this preprint (which was not certified by peer review) is the author/funder. All rights reserved. No reuse allowed without permission.

113

A

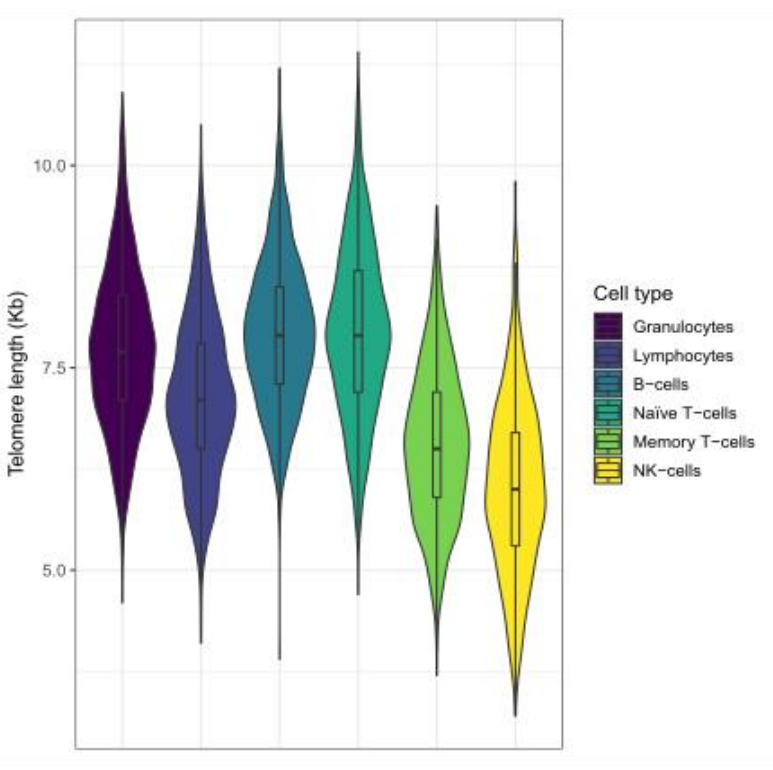

B

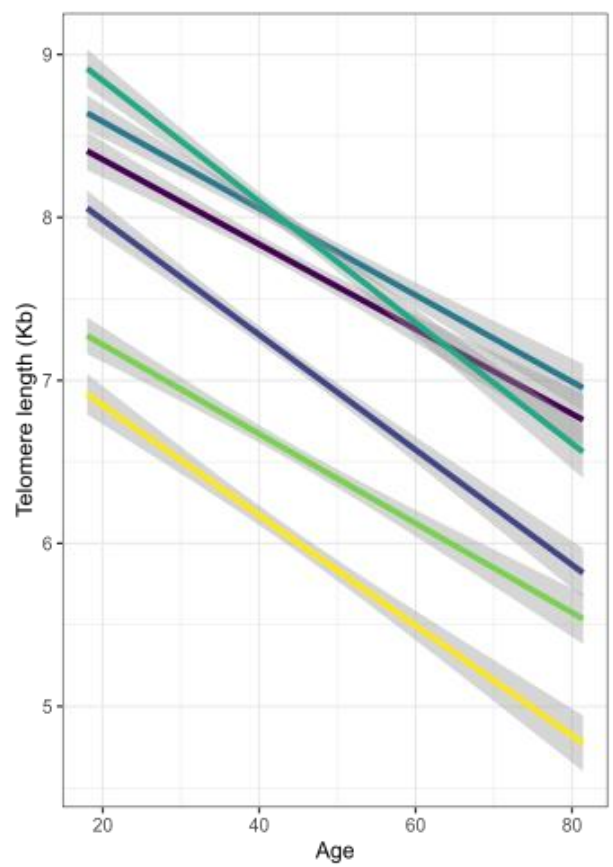

D

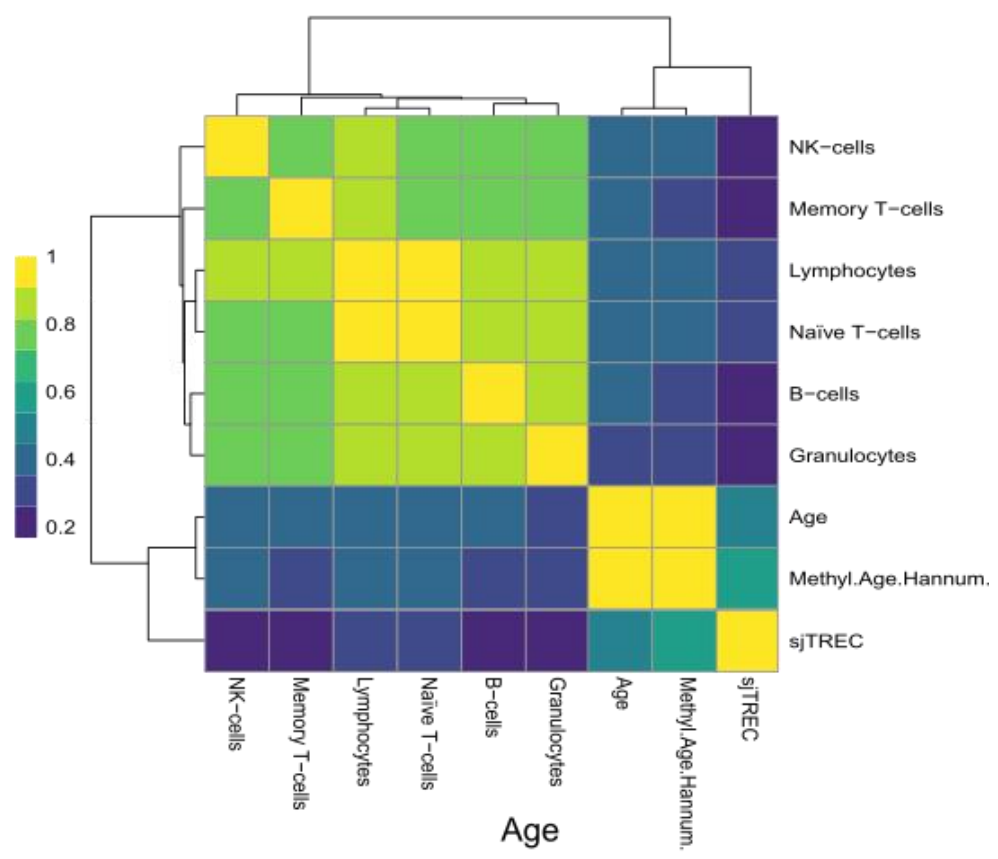


116 Figure 1. Telomere measurements in six blood cell types. A. Distribution of telomere length by cell type. B.

117 Average trend line of telomere length. Grey shading indicates 95\% confidence interval. Length decreases with age

118 in all six cell types. Colour indicates cell type in both (A) and (B). C. Sexual dimorphism of telomere length (shown

119 in lymphocytes). Trend line indicates average length per age. Grey shading indicates $95 \%$ confidence interval. D.

120 Correlation (Spearman's rho value) between the absolute value of telomere lengths, chronological age (Age),

121 Hannum-based methylation age and sjTREC qPCR relative expression.

123 Genetic contribution to telomere length

124 To explore to what extent genetics can explain variation in telomere length, we first performed a heritability 125 analysis. We used genotype data to infer genetic relations and fit a GREML model, while controlling for age and 126 sex. This analysis provides an estimate of the total telomere length variability attributable to the additive effects of 127 common genetic variants. The results show a median SNP-based heritability of $45 \%$, with a maximum of $51 \%$

128 (naïve T-cells) and a minimum of 19.6\% (NK-cells). Although the standard errors of the estimates were high, with a 129 median of $22 \%$ [Figure $\mathbf{2 A}$ ], the heritability estimate falls in a similar range to those of previous reports ${ }^{37-39}$ and the 130 corresponding P-values were below 0.05 for all cell types except NK-cells $(\mathrm{p}=0.15)$. This result supports the idea 131 that genetic factors contribute significantly to telomere length and can partially explain the inter-individual 132 variability. The higher genetic contribution to naïve T-cell telomere length that we observe might be explained by 133 the fact that these cells reflect less environmental influence. On the other hand, environmental factors will impact 134 telomeres in memory T-cells, including NK-cells, because antigen-mediated clonal expansion of these cells is 135 typically triggered by environmental factors (e.g. infections), and thus telomere length will decrease due to the 136 number of replications.

137 To further explore the genetic contribution to telomere length, we performed a genome-wide association study 138 (GWAS) of $~ 7.5$ million genotyped or imputed SNPs on telomere length in each cell type. First, we tested if we 139 could replicate previously published loci associations. For this, we used the 20 genome-wide significant loci from a 140 recent meta-analysis published by Li et $\mathrm{al}^{15}$. Of the 17 published SNPs for which we also had information, six were 141 replicated in at least one cell type with a p-value $<0.05$ and consistent allelic direction [Supplementary Table 2.1].

142 Similarly, we could replicate 6/12 European-associated genome-wide significant loci from a large trans-ethnic

143 study $^{16}$ [Supplementary Table 2.1]. We then looked at de novo associations and identified one significant 
144 association after applying permutation-based false discovery rate (FDR) correction: The A allele of rs33961405

$145(1000 \mathrm{G}$ EUR allele frequency $=0.49)$ located in an intron of the telomerase reverse transcriptase $($ TERT $)$ gene was

146 associated with decreased telomere length of T-cells (effect size $=-0.19, \mathrm{SE}=0.03$ ) [Figure 2B][Supplementary

147 Figure 3] (summary statistics of associations $\mathrm{p}<1 \times 10^{-5}$ presented in [Supplementary Table 2.2]). Other genetic

148 variants located near the TERT gene were previously found to affect leukocyte telomere length; however,

149 rs33961405 is novel and is in moderate linkage disequilibrium (LD) with a previously published lead SNP in TERT

$150 \quad(\text { rs2736100, LD r2 }=0.47 \text { in } 1000 \mathrm{G} \text { EUR data })^{12,13}$.

151 To reproduce previous GWAS associations on the individual SNP level, we computed a Polygenic Risk Score (PRS)

152 from a large GWAS on telomere lengths ${ }^{15}$ (see Methods) and correlated it to our telomere length data. This analysis

153 identified highly significant positive associations. The strongest genetic associations were seen in cell types with

154 longer telomeres: naïve T-cells (linear model $(\mathrm{lm})$, effect estimate $\left.=1.5, \mathrm{p}=7.61 \times 10^{-13}\right)$ and B-cells $(\mathrm{lm}$, effect

155 estimate $\left.=1.19, \mathrm{p}=6.78 \times 10^{-10}\right)$. Cell types with shorter telomeres, memory T-cells $(\mathrm{lm}$, effect estimate $=0.89, \mathrm{p}=$

$\left.1567.47 \times 10^{-6}\right)$ and NK-cells ( $\mathrm{lm}$, effect estimate $\left.=0.97, \mathrm{p}=1 \times 10^{-5}\right)$, showed weaker associations of the genetic

157 determinants. An exception to this trend was granulocytes, which showed a similar association range to B-cells and

158 NK-cells $\left(\mathrm{lm}\right.$, effect estimate $\left.=0.95,6.02 \times 10^{-6}\right)$, despite having one of the longest average telomere lengths. 
A

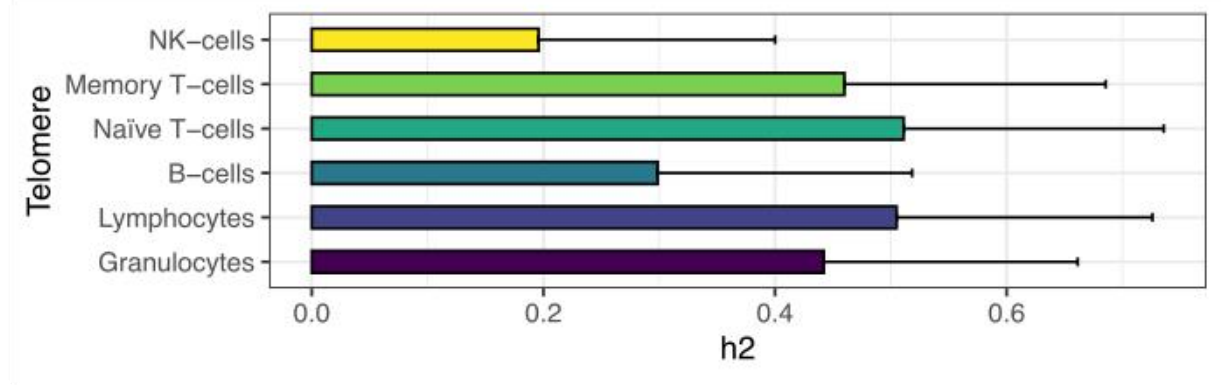

B
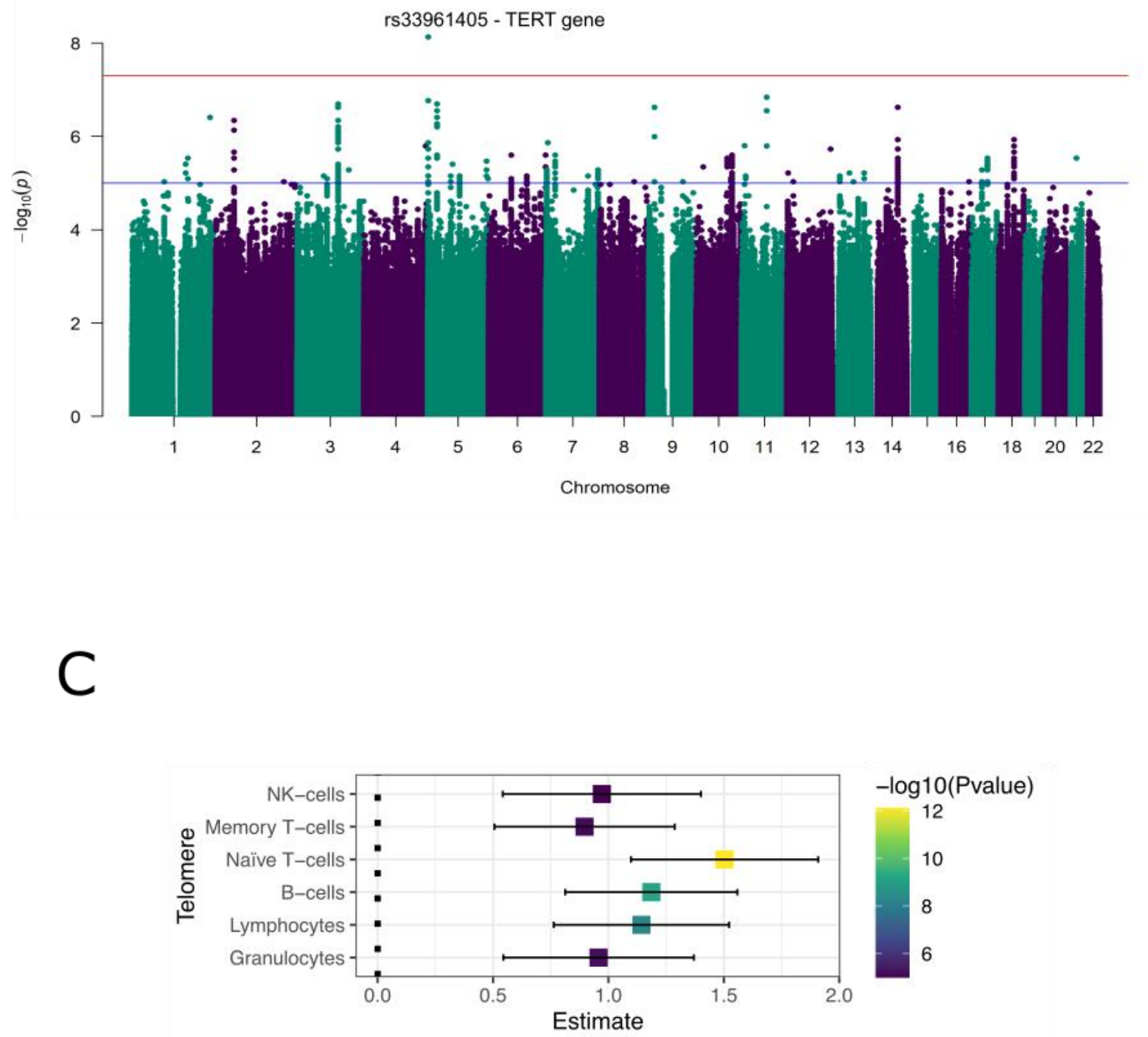
160 Figure 2. Genetic determinants of telomere length. A. Heritability estimation using a GREML model on the 161 genotyped SNP data, from which a kinship matrix was estimated using GCTA ${ }^{40}$. The kinship matrix was used in a

162 mixed model to estimate the variation explained by the genetic random effect. Units of heritability are represented as

163 h2. B. Manhattan plot of genome-wide associations to telomere length from all cell types. Blue line indicates a

164 suggestive significance threshold of $1 \times 10^{-5}$. Red line indicates the standard genome-wide threshold of $5 \times 10^{-8}$. The

165 novel association with TERT, rs33961405 (5:1277577:G:A GRCh37), is labelled with its corresponding rsid. C.

166 Correlation of the Polygenic Risk Score (PRS) of telomere length ${ }^{15}$ and telomere length of different cell types. Each

167 square represents the estimated effect of the PRS in the cell type. Bars indicate $95 \%$ confidence interval.

\section{Telomere ancestry - parental age and smoking contribute to an individual's telomere length}

170 We exploited the extensive phenotypic information available for LLD study participants to uncover which

171 environmental factors are correlated with telomere length. The phenotypic information consists of 90 different

172 parameters, including blood parameters (e.g. leukocyte counts), anthropometric measurements (e.g. BMI),

173 physiological parameters (e.g. blood pressure), various pre-existing diseases (e.g. hypertension or cancer) and

174 lifestyle factors (e.g. smoking), as well as parental phenotypes and habits including parental diseases, smoking and 175 age at participant's birth [Supplementary Table 3].

176 To associate telomere length with different phenotypes, we built a linear model using telomere length as the 177 dependent variable and the standardised phenotype measurement as the regressor, while controlling for age and sex.

178 Of the non-genetic factors, blood cell counts were strongly associated with telomere lengths (Supplementary Table

179 4.1). Since cell counts might act as a confounder for other associations (as cell types have different telomere length

180 and thus might confound the observed inter-individual differences), we included cell counts as covariates in the 181 model. Using this new model, we identified 37 associations of 12 phenotypes with telomeres of any cell type using 182 an FDR < 0.05 threshold (summary statistics can be found in Supplementary Table 4.2).

183 Several parental factors were consistently associated with telomere lengths in different cells. Smoking phenotypes 184 such as 'any parent smoking', 'father smoking during your childhood' and 'mother smoking during pregnancy' were 185 negatively associated with the telomere lengths of almost all cell types (with the exception of NK-cells). 'Age of 186 father when you were born' and 'age of mother when you were born' were positively associated with the telomere 187 lengths of four cell types. In a model combining both paternal and maternal age (on an 11 penalisation, see 
188 Methods), we found paternal age to shrink to near 0 while maternal age was kept constant (with the exception of granulocytes), highlighting that the effect of maternal age on telomere length is independent of paternal age.

190 Significant negative correlations with smoking were only observed with the father, both parents in combination and 191 the mother during pregnancy. However, all cell types also showed nominally significant associations with maternal 192 smoking. Maternal smoking associations showed weaker effect sizes than paternal (Im, 0.06 average difference, SE: $\left.1930.01, \mathrm{p}=3.2 \times 10^{-4}\right)$ and fewer participants had mothers who smoked than fathers who smoked (674 fathers vs. 381

194 mothers), factors which together may explain why maternal smoking did not reach FDR significance. Paternal and

195 maternal smoking were, however, shown to have additive effects: A model that included a numeric variable

196 describing the number of parents who were smokers showed stronger associations than binary smoking phenotypes

197 (father, mother, or any parent).

198 We further analysed the associations to other available smoking phenotypes. Here we found a consistent nominally 199 significant negative effect of 'father smoking', 'mother smoking' and 'mother smoking during pregnancy' in all the 200 cell types tested. In addition, passive smoking during an individual's lifetime also influenced telomere length, with 201 the factor "do people smoke near you at work" associated to shorter telomeres in 5/6 cell types. Conversely, current 202 smoking of the participant did not reach nominal significance in any cell type [Supplementary Figure 4A].

203 In addition, we found four negative associations with participant BMI and three with participant waist

204 circumference. These negative associations with BMI and waist circumference could be driven by their correlations

205 to other phenotypes. We therefore explored the association of BMI with other phenotypes in a larger cohort of

20610,000 participants from the same population ${ }^{41}$ and observed a positive correlation between BMI and parental

207 smoking (Pearson, $\left.\mathrm{p}<4.78 \times 10^{-6}\right)$ and a negative correlation between BMI and parental age $\left(\right.$ Pearson, $\left.\mathrm{p}<9.15 \times 10^{-7}\right)$.

208 After accounting for potential confounding effects of parental age and smoking habits, the associations between

209 telomere length and BMI phenotypes remained significant [Supplementary Figure 4B]. This observation supports

210 the conclusion that the associations of telomere length to BMI are not driven by the confounding effects of parental

211 age or smoking.

212 In addition, we found one cell-specific (having one cell type below FDR < 0.05) positive association with poorly

213 healing wounds (granulocytes) and three cell-specific negative associations with blood alpha-1 antitrypsin (AAT) 
214 (Memory T-cell), pulse rate (granulocytes) and weight (B-cells) [Figure 2A] (summary statistics in Supplementary

\section{Table 4.2).}

216 Finally, we assessed how much of the variation in telomere length not attributable to participant age could be

217 explained by intrinsic, parental and genetic factors. For environmental factors, we included all associated factors

218 with an FDR $<0.05$ in at least two cell types. Genetic factors were addressed using PRS from a recently published

219 telomere length GWAS $^{15}$, see Methods. We fitted four nested models and estimated the added variability (R2)

220 explained by each (see Methods) [Figure 3B]. This revealed that most of the variability is explained by the addition

221 of sex, BMI, waist circumference and cell counts (from 3.2 to $8 \%$ ), depending on the cell type. The addition of

222 parental phenotypes (parental age and smoking) added less information (0.8\%), on average, than the multiple

223 intrinsic factors. Finally, the contribution of genetics (average 3.7\%) was lower than that of intrinsic factors (average

$2245.9 \%$, considering sex as an intrinsic factor rather than a genetic one) in most cells, which is in line with the larger

225 impact of environmental effects that we observed in the heritability analysis.

227 Epigenetic changes may mediate the effect of parental phenotypes on telomere length

228 It has been proposed that maternal and paternal phenotypes, such as smoking habits and age at pregnancy, may 229 affect a child's phenotypes by inducing changes in methylation levels ${ }^{42-47}$. Several studies have reported an effect of

230 parental age and smoking on the "methylation age" of the child ${ }^{43,44,46,48}$. We therefore investigated whether the effect

231 of parental phenotypes on telomere length could be mediated by changes in methylation levels. We first performed a

232 GWAS of parental smoking and age with DNA methylation levels available for the same samples $(\mathrm{N}=749)$. This

233 resulted in 19 genome-wide significant associations of methylation probes with parental phenotypes at a Bonferroni-

234 corrected $\mathrm{p}<0.05$ (summary statistics in Supplementary Table 5). Combining these results with the associations of

235 parental phenotypes with telomere length $(\mathrm{p}<0.05)$ and of telomere length with methylation $(\mathrm{p}<0.05)$, we

236 performed mediation analysis for the 17 resulting triplet associations. This analysis identified four triplets that

237 showed significant mediation of the parental phenotype effect on telomere length through methylation of various

238 probes (see Methods) [Supplementary Table 5]. All these significant mediation results were for the age of mother,

239 even though we tested fewer triplets for maternal (6 triplets) than paternal age (11 triplets). Maternal age effects thus

240 appear more likely to be mediated by methylation than paternal age effects. 
241 One interesting example of this methylation mediation is for the SRY-related HMG-box gene SOX11. This gene is a

242 known transcription factor and proliferation gene that plays an important role in embryonic development, cell fate

243 determination and cancer. Our results indicate that the positive association of maternal age at birth with telomere

244 length in NK-cells may be mediated by decreased methylation of CpG islands located in the promoter of SOXI1

245 (mediation $\mathrm{p}<2 \times 10^{-16}$ ), with up to $28 \%$ of the effect mediated through methylation of SOX11 [Figure 3C]

246 [Supplementary Table 5]. 


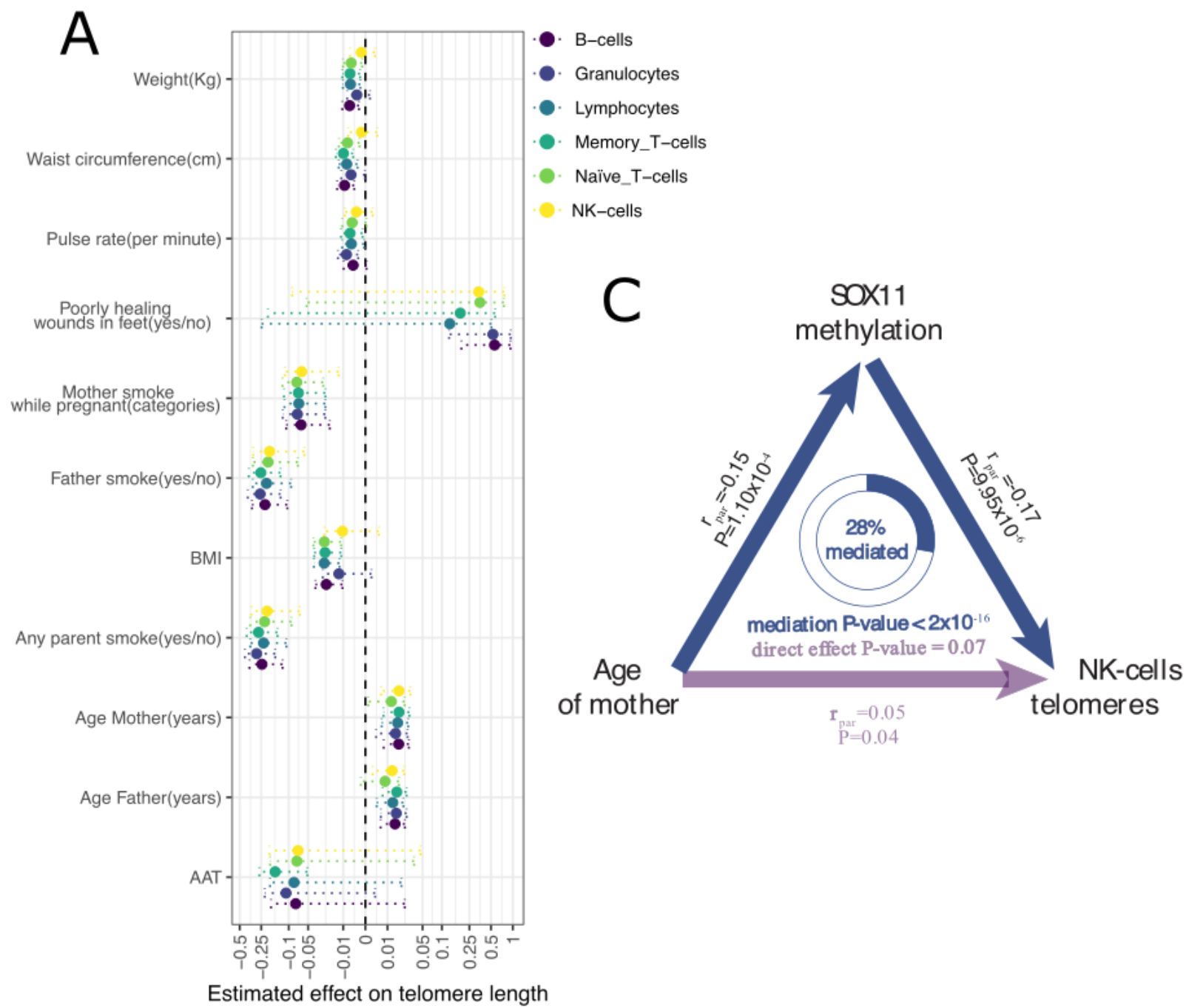

B

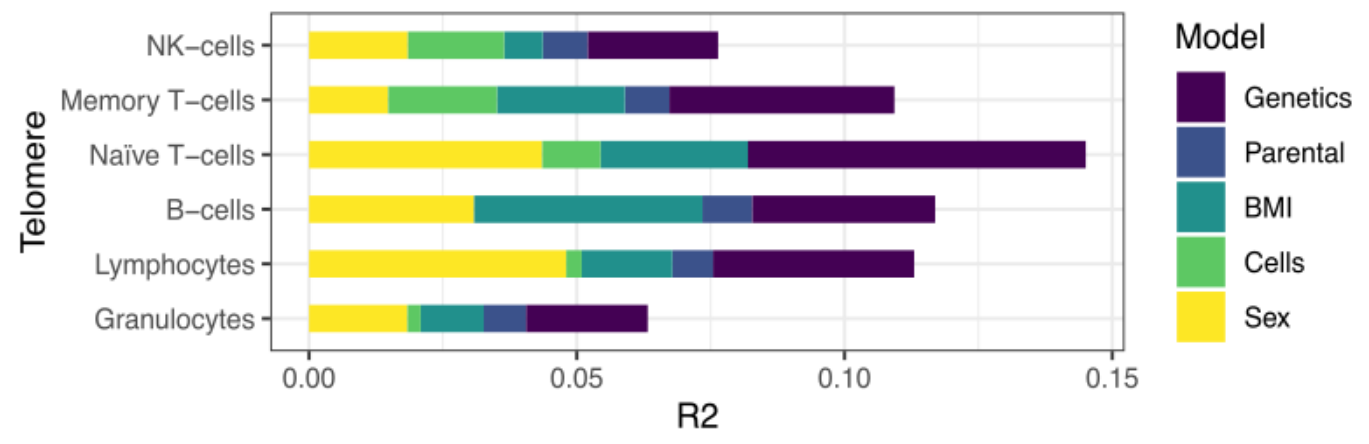


248 Figure 3. Non-genetic factors contribute to telomere variation. A. Phenotype effect on telomere lengths of

249 different cell types (with at least one significant association). Dashed lines show 95\% confidence interval (estimate

$250 \pm 2 \mathrm{xSE}) . \mathrm{X}$-scale is symmetrical log-transformed (denominator constant $=-2)$. BMI: Body mass index, AAT:

251 Alpha-1 Antitrypsin Test. B. Total variance of the telomere length explained after removing the effect of age. Only

252 phenotypes associated with at least two cell types (FDR < 0.05) are used. Colour indicates the different partitions of

253 variability. C. Mediation effect of methylation of SOX11 in the maternal age effect on NK-cell telomere length

254 variability.

Telomere length changes show cell-type-specific associations with gene expression level

257 To study the relationships between telomere length variation with gene expression changes, we used single-cell

258 RNA-sequencing (scRNA-seq) data generated on cryopreserved peripheral blood mononuclear cells (PBMCs) from

25962 LLD donors, for which telomere length on six cell types was measured ${ }^{49}$. To classify cells, we combined the high

260 resolution cell-type-annotations by Azimuth $^{50}$ to closely reflect the resolution of the Flow-FISH annotations (i.e.

261 naïve and memory CD4T and CD8T cells, NK- and B-cells) (see Methods) (Supplementary Table 6). First, we

262 confirmed that the subset of 62 LLD donors had similar telomere length distributions to the entire study population

263 (1,046 LLD donors) (Supplementary Figure 5). We then performed telomere length differential gene expression

264 analyses at single-cell resolution (sc-DEA) by selecting the matched telomere length measurement and gene

265 expression level for each of these cell types (see Methods) (Supplementary Table 6). These analyses revealed

266 DNAJA1 to be positively associated with telomere length in memory CD8T cells (effect size $=0.05$ log-fold change

267 (LFC) per telomere length unit, FDR = 0.03) (Supplementary Table 7.1). DNAJAl encodes a heat shock protein 70

268 co-chaperone that was previously reported to bind telomeres in a study that used in vivo cross-linking, tandem

269 affinity purification and label-free quantitative mass spectrometry ${ }^{51}$.

270 Because we only identified one differentially expressed (DE) gene, we wondered whether this could be due to

271 insufficient statistical power. To address this, we performed differential gene expression analysis on T-cells

272 combining multiple cell types together (Methods) while controlling for cell type annotation (Supplementary Table

273 6). This strategy increased the number of cells per donor and thus statistical power. However, in contrast to

274 analysing each cell type separately, genes identified with this combined strategy will likely have a similar

275 association with telomere length across cell types. We identified 97 unique DE genes, one in CD8T cells, 44 in 
CD4T cells and 91 in all T-cells (Figure 4) (Supplementary Table 7.2, Supplementary Figure 6), including the

DNAJA1 association reported in our previous analysis

Next, we explored potential mechanisms explaining the telomere length associations with expression in the set of 97 genes. Several mechanisms have been described by which telomere length may affect gene expression levels. The first is the telomere position effect (TPE) ${ }^{26}$. This mechanism results in decreased repression of genes located in the subtelomeric region when telomeres shorten ${ }^{27}$ and in subsequent overexpression, which is a process associated with CpG-methylation ${ }^{29}$. To determine whether our sets of DE genes were influenced by TPE, we tested whether there was an enrichment of DE genes in the subtelomeric region (<4Mb from chromosome end) (Supplementary Table

8). In CD4T cells, we found a significant enrichment of genes positively $(5 / 19, \mathrm{p}=0.007)$ but not negatively associated $(0 / 24, p=1)$ associated with telomere length (Supplementary Figure 7). We did not observe any enrichment in all T-cells $(8 / 45, p=0.05$ for positively associated and $4 / 45, p=0.58$ for negatively associated genes) (Supplementary Table 8). The enrichment we observe in the CD4T cells is opposite to what we expected, i.e. we expected to identify genes that were negatively associated with telomere length as a result of the lost repression due to shortening. Our contrasting findings suggest that other mechanisms might be at work in the associated genes.

A second mechanism by which telomere length may affect gene expression levels occurs through a telomere

292 position effect over long distances (TPE-OLD). Such long-distance gene expression regulation was shown to be the 293 result of telomeres forming chromatin loops with enhancer and repressor regions of genes within $10 \mathrm{Mb}$ of

294 chromosome ends ${ }^{28}$. These interactions are dependent on telomere length but are not linearly related with shortening 295 of the telomeres. We did not find any enrichment within our DE gene sets for genes acting through a potential TPE296 OLD mechanism (i.e. 4-10Mb from chromosome end) in either T-cells (4/45 DE genes positively associated with 297 telomere length, $p=1 ; 5 / 45$, DE genes negatively associated with telomere length, $p=0.8)$ or CD4 cells $(1 / 19$ DE 298 genes positively associated with telomere length; $p=0.7 ; 4 / 24 \mathrm{DE}$ genes negatively associated with telomere length, $299 \mathrm{p}=0.52)($ Supplementary Figure 7) (Supplementary Table 8). However, the generalised linear mixed model

300 (GLMM) we used for sc-DEA assumes linearity and therefore might not allow us to properly test this hypothesis, 301 which assumes non-linear relations.

302 Since many of the identified DE genes did not fall into the TPE or TPE-OLD categories, other unknown effects on 303 gene expression through telomere length may be at play or, conversely, the expression of specific genes may affect 
304 telomere length. For several DE genes, we found additional evidence for a telomere connection in literature (Figure

305 4) (Supplementary Table 8). Firstly, three of our hits (DNAJA1, EEF1A1 and RPL29) were previously reported in

306 a screen for novel telomere binding proteins, indicating their direct involvement in telomere maintenance ${ }^{51}$.

307 Secondly, CpG methylation of NR4A2 was previously found to be associated with telomere length in whole blood ${ }^{29}$.

308 NR4A2 is involved in T-cell maintenance through regulation of Treg suppressor functions and repression of aberrant

309 Th1 induction ${ }^{52}$. The third line of gene-telomere interaction evidence is the overlap with leukocyte ageing-

310 associated genes that were previously identified in whole blood after a deep correction for cell type composition (8

311 out of 84 testable genes overlapped; odds ratio $\left.=3.3, p=6 \times 10^{-3}\right)^{53}$. Finally, four genes also overlapped with the

312 GenAge human database, the core Human Ageing Genomic Resources (HAGR) database composed of >300 human

313 ageing-related genes ${ }^{54}$. These four were related to immunosenescence ${ }^{55,56}(I L 7 R)$, cellular senescence ${ }^{57,58}(M Y C)$ and

314 longevity ${ }^{59-62}$ (EEF1A1 and MT-CO1), in line with telomere shortening being a well-known hallmark of both

315 cellular senescence and organismal aging ${ }^{63,64}$. However, we did not find a significant overlap between the genes we

316 identified and the ones reported in that study (odds ratio $=1.4, \mathrm{p}$-value $=0.53$ ).

317 After exploring individual gene associations, we wondered if the identified DE genes belonged to similar functional

318 pathways and could thus highlight the biological interplay between telomeres and gene expression. To explore this,

319 we performed a functional enrichment analysis within the different sets of DE genes. In CD4T cells, the JAK-STAT

320 signalling pathway was negatively associated with telomere length (enrichment ratio $=18.63, \mathrm{FDR}=4 \times 10^{-3}$ )

321 (Supplementary Table 9). This pathway has previously been associated with telomerase regulation in haematologic

322 malignancies ${ }^{65}$ and immunosenescence ${ }^{56}$. Among the DE genes involved in the JAK-STAT signalling pathway,

323 interleukin-7 receptor (IL7R) plays a critical role in lymphoid cell development ${ }^{66,67}$, and its gene expression network

324 has been proposed as a potential biomarker for healthy aging ${ }^{55}$. In all T-cells, we identified 26 pathways enriched

325 among the genes negatively associated with telomere length, many of them related to translation, including peptide

326 chain elongation, eukaryotic translation elongation or termination and initiation, among others (Supplementary

327 Table 9). Previous genetic screens in yeast ${ }^{68-71}$ and Arabidopsis thaliana ${ }^{72}$ identified ribosome biogenesis as one of

328 the largest gene categories linked to telomere length. On top of that, in human fibroblasts, cellular senescence

329 triggered through telomere shortening can diminish ribosome biogenesis, resulting in rRNA precursors and

330 accumulation of ribosomal proteins (such as RPL29, which we found to decrease expression with telomere length in

331 T-cells $)^{73,74}$. In addition, our set of negatively enriched pathways in T-cells also revealed the nonsense-mediated 

specific mRNAs that are important for telomere functions ${ }^{76}$.

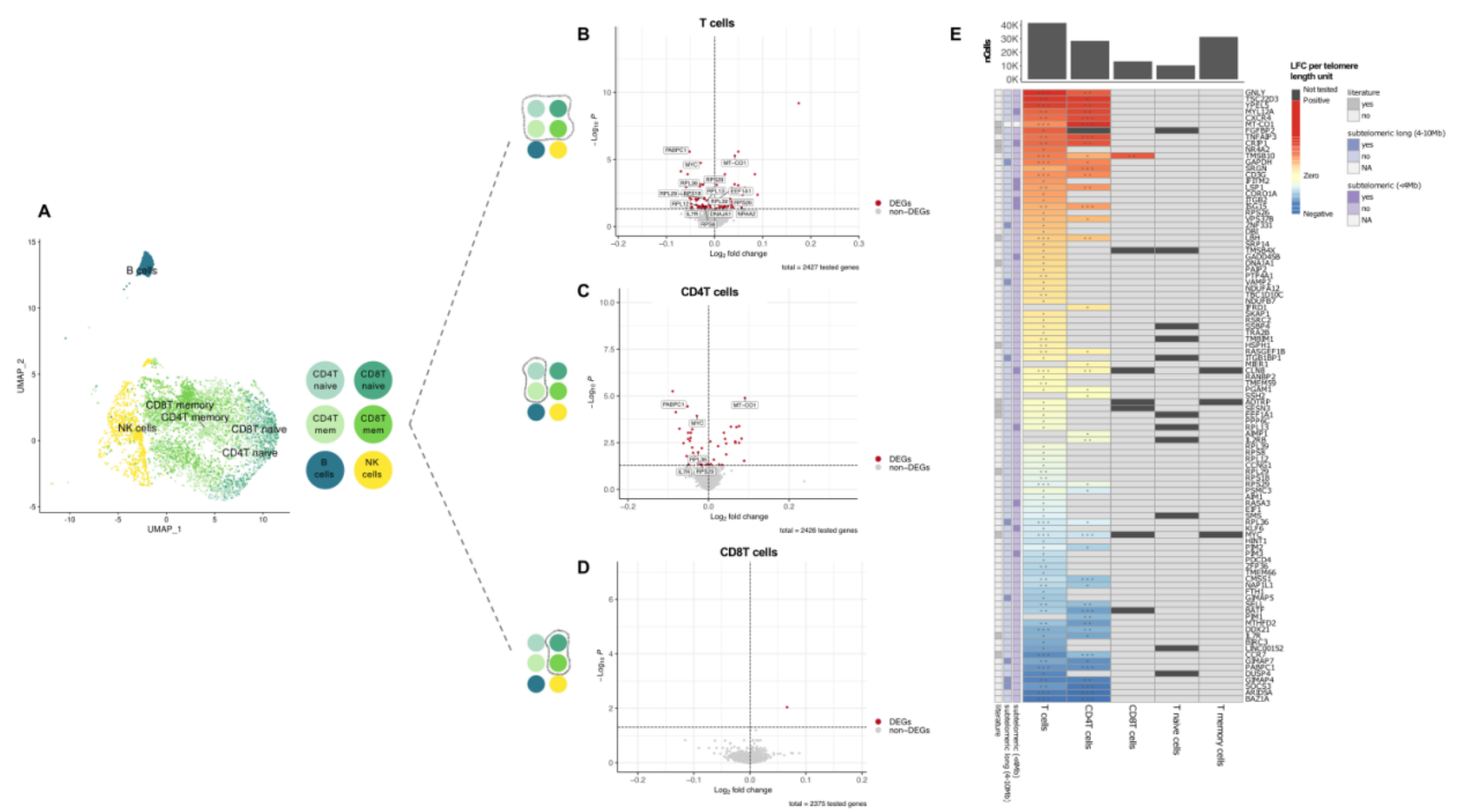

Figure 4. Differential gene expression changes with telomere length across cell types. A. UMAP plot of the cells

336 from the subset of 62 LLD donors with both scRNA-seq and Flow-FISH telomere length data. The cells are coloured by the cell type classification that closely reflects the resolution of the Flow-FISH annotations (i.e. naïve and memory -mem- CD4T and CD8T cells, NK- and B-cells). For visualisation purposes, we down-sampled each of Azimuth's predicted cell types to 500 cells. B, C and D. Volcano plots showing the results of DE approach II in T-, CD4T and CD8T cells, respectively. For DE approach II, we combined multiple cell types together (in A) in the

341 same analysis (i.e. combining all T, all CD4T, all CD8T, all naïve T and all memory T-cells). DEGs with telomere 342 length are represented in red. Non-DEGs are represented in grey. Labels correspond to the DEGs mentioned in the

343 text. E. Heatmap of log-fold change (LFC) per telomere length unit for the set of 97 unique DEGs identified in T-,

344 CD4T and CD8T cells. Non-DEGs are shown in light grey. Genes not tested (i.e. those below 10\% expression cut-

345 off) are shown in dark grey. The significance level of the DEGs corresponds to the following arbitrary FDR

346 thresholds: FDR $<0.001(* * *)$, FDR $<0.01(* *)$ and FDR $<0.05(*)$. The genes are sorted by their average LFC across

347 cell types. The row annotation bars show whether the genes are located at the subtelomeric $(<4 \mathrm{Mb})$ or subtelomeric

348 long $(4-10 \mathrm{Mb})$ region and if the genes were previously reported in any of the following studies: Pellegrino-Coppola 
et al., 202153, Tacutu R et al., 201854, Buxton JL et al., 2014 ${ }^{29}$ or Nittis T et al., $2010^{51}$ (Supplementary Table 8).

The distance to the telomeres was not calculated for the mitochondrial gene (MT-CO1) (subtelomeric $(<4 \mathrm{Mb})$ and subtelomeric long $(4-10 \mathrm{Mb})=\mathrm{NA})$. The column annotation on the bar plot shows the total number of cells (nCells)

352 per cell type.

\section{3}

354

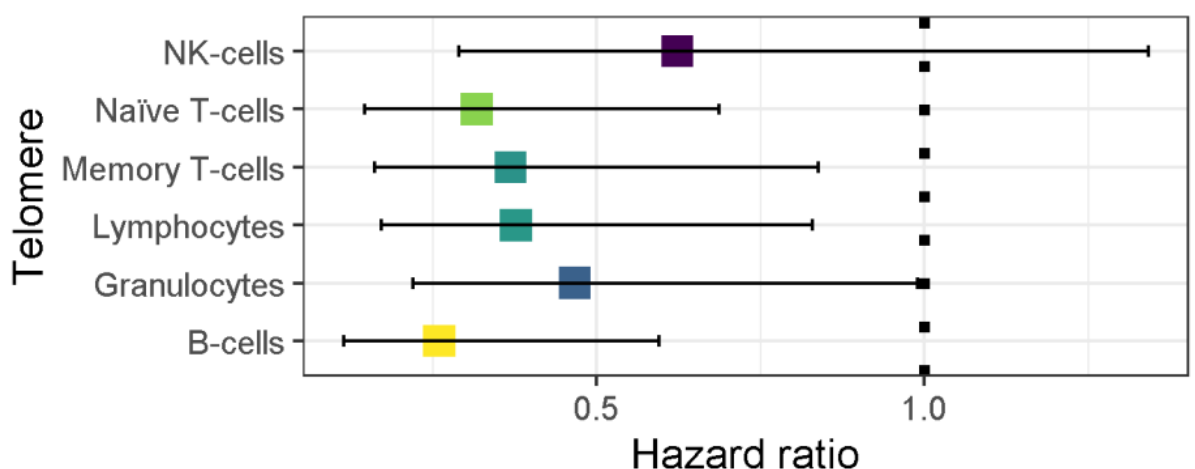

\section{$-\log 10($ Pvalue)}

\section{5}

2.0

Figure 5. All-cause mortality risk of telomere length. Y-axis represents the hazard ratio of all-cause mortality estimated by a Cox regression on each cell type's telomere length. Bars indicate estimated $95 \%$ confidence interval.

\section{Discussion}

368 This work presents the largest study to date on telomere length variation in a population cohort based on cross-

369 sectional Flow-FISH ${ }^{33}$ measurements. We measured telomere lengths in six immune-related cell types from 1,046 participants from the northern Netherlands LLD cohort ${ }^{32}$. We investigated the effect of genetics and 90 phenotypes, 
371 including parental factors and a wide range of general and environment factors, on telomere length. This identified

372 the most important factors that influence telomere length and, based on our findings, we propose potential

373 mechanisms of action for some of these factors. Finally, we identified cell-type-specific transcriptional modules

374 related with immuno-senescence that might explain the downstream effects of telomere length attrition.

375 Previous studies of telomere length variation in population cohorts have mainly relied on classical terminal

376 restriction fragment measurements ${ }^{77}$, qPCR-based methods ${ }^{78,79}$ or computational methods ${ }^{16}$ to measure telomere

377 length. In the current study, we used Flow-FISH in a large epidemiological setting. Flow-FISH allows accurate

378 measurements of the average telomere length in different cell types ${ }^{80}$. This allowed us to observe the distribution of

379 telomere length in different cell types in addition to the more commonly studied telomere length of total leukocyte

380 population ${ }^{15,18,19}$. Our observations reveal a similar trend of telomere length decrease with age and a high correlation

381 in telomere length between different cell types (Pearson correlation $\mathrm{r}$ minimum 0.70, mean 0.81, maximum 0.94).

382 These finding overlap with previously published between-tissue telomere length correlation ranges (leukocyte, skin,

383 skeletal muscle and subcutaneous fat, Pearson $\mathrm{r}$ from 0.76 to 0.88$)^{81}$ but show a smaller range than those reported in

384 post-mortem tissues (highest Pearson $r=0.40$ between transverse and sigmoid colon) ${ }^{20}$. Nevertheless, it must be

385 noted that measurements were carried out using Southern blot in ${ }^{81}$, whereas the measurements in ${ }^{20}$ where Luminex-

386 based. These findings agree with previous studies that indicated that despite having different telomere lengths in

387 adults, the overall effect of age on telomere length is similar in different cell types ${ }^{81}$. Additionally, our results show

388 high reproducibility with respect to a previous Flow-FISH population study in a North American cohort 9.

389 The average telomere length in each cell type is a complex phenotype that reflects cell-type-specific, genetic,

390 intrinsic and environmental effects. Many previous studies have tried to estimate the role of genetics in explaining

391 the variation of telomere length in humans ${ }^{18}$, producing a wide range of estimates. These differences suggest there

392 are potential environmental effects confounding the heritability estimates. For instance, sibling-based studies ${ }^{17,38,82}$

393 have the disadvantage of not being able to distinguish between genetics and early life or prenatal exposures. In our

394 study, which more closely resembles approaches that estimate "chip-based" heritability in unrelated individuals ${ }^{83,84}$,

395 we estimated narrow sense heritability in six different cell types based on a mixed-model approach using sample-to-

396 sample genotype kinship estimations $(\mathrm{GREML})^{40}$. One advantage of this study is that LLD participants were

397 specifically selected to have a common ancestry but not be highly related among each other, meaning that the

398 GREML estimation should not be biased by those factors. After accounting for age and sex, our GREML estimation 
indicates a median heritability of $40 \%$, which is in the same range as previously reported ${ }^{85}$. However, our estimate does not include the $\mathrm{X}$ or $\mathrm{Y}$ chromosomes, which might play a role in telomere heritability ${ }^{86}$. In addition, the

401 relatively small sample size of our study $(1,000)$ hampers the accuracy of our estimation, and we observe large

402 standard error intervals. Nevertheless, the heritability levels that we find are indicative of a large amount of telomere

403 variability being caused by environmental factors, which contrasts with other studies that identified extremely high

404 telomere heritability $\left(0.7^{19}, 0.78^{82}, 0.81^{87}\right)$. In our GWAS analysis, we identified one intronic SNP in the TERT gene

405 that was associated at $5 \times 10^{-8}$ and in moderate $\operatorname{LD}(0.47)$ with previously reported associations in this locus. The

406 GWAS analysis in our cohort was limited by our relatively small sample size (911 individuals), but PRS scores from

407 a recent large telomere GWAS ${ }^{15}$ indicate strong association of previously identified genetic variants with telomere

408 length of all cell types.

409 Despite the on-going efforts to understand the effect of genetics on telomere length ${ }^{12-16}$, a large fraction of

410 unexplained variability remains, which may be attributed to environment. We therefore investigated the relation of

41190 phenotypes with telomere length. This analysis pointed to a role for parental phenotypes in the telomere

412 variations in their children. Higher paternal age has previously been associated with longer telomere lengths in

413 humans ${ }^{19}$. Our results agree with this but also identify a maternal age effect on telomere length. Given the high

414 correlation between paternal and maternal age $(r=0.77)$, the maternal age effect we observe might be confounded

415 by paternal age. It is known that sperm telomere length increases with age, whereas telomere length in somatic

416 tissues, including leukocytes, diminishes with age ${ }^{88,89}$ providing a potential explanation for longer leukocyte

417 telomere length in offspring of older fathers. This effect might even be additive over consecutive generations ${ }^{90}$. In

418 addition to parental age, we identified a novel telomere association with parental habits in which parental smoking

419 was negatively associated with telomere length. This effect could either be prenatal or early life.

420 Additionally, even with the possibility of being caused by common genetic architecture, parental effects in offspring

421 are more plausibly caused by a mechanism other than genetics, such as epigenetic modifications. We therefore

422 tested whether methylation might mediate the effect of parental phenotypes such as age and smoking on telomere

423 length in their children. The effect of maternal age, and not paternal age, on telomere length was mediated through

424 methylation of several genes, suggesting that the maternal age effect is not completely confounded by paternal age.

425 Here we highlighted the mediation of telomere length in NK-cells via the methylation of SOX11. It is not clear how

426 SOX11 methylation may lead to telomere shortening, as SOX11 is mostly expressed during embryonic development. 
427 Previous studies have shown that promoter hypermethylation of SOX11 inhibits SOX11 expression in cancer cells ${ }^{91}$.

428 In addition, several studies have shown an anti-senescent effect of SOX4, which belongs to the same SOXC group as

$429 \operatorname{SOX} 11^{92}$. It is important to stress, however, that this is a proposed mechanism, and that experimental validation will

430 be needed to resolve the nature of such associations.

431 Apart from parental factors, we identified that women have longer telomeres than men, as previously identified ${ }^{86,93}$.

432 In addition, BMI was associated with shorter telomeres ${ }^{20,94}$. However, we did not observe other previously reported

433 associations, such as with participant smoking habits ${ }^{20,95}$. Combining all identified intrinsic, parental and genetic

434 factors other than age with significant effects at least in two cell types, we estimate that only $10 \%$, on average, of the

435 observed telomere length variability can be explained, with only about 3.7\% attributable to genetics (without

436 accounting for the genetics factors that cause sex differences). These findings show that environmental and intrinsic

437 effects have a greater impact than genetics on telomere length variation. Nevertheless, it is worth noting that the

438 model applied in this study does not consider interactions between the different layers of information, where

439 genetics could potentially impact the effects of the other environmental features. In addition, we are only

440 considering highly associated additive SNPs and no other possible epistatic relationships, which means we are

441 probably only setting a lower bound on the amount of variability that we can account for.

442 Our single-cell transcriptomic analyses identified a set of 97 unique genes that are significantly associated with

443 telomere length across T-cell types. Three of them (DNAJA1, EEF1A1 and RPL29) were previously revealed as

444 telomere binding proteins ${ }^{51}$, indicating that our approach captures genes directly involved in telomere length

445 dynamics. Moreover, our study provides additional insight into the direction of effect. For example, we found

$446 \quad R P L 29$ to be negatively associated with telomere length, which is in line with previous studies describing

447 accumulation of RPL29 as a biomarker of senescence ${ }^{73}$. Looking at the broader context, we observed functional

448 enrichment of genes involved in translation and the NMD pathway within the gene set negatively associated with

449 telomere length in T-cells, which might have important physiological consequences.

450 Even though some of the telomere length-associated genes were located near the telomere ends (i.e. 12 and 9 DE

451 genes that fall $<4 \mathrm{Mb}$ and between $4-10 \mathrm{Mb}$ from the telomere ends, respectively), in general, our differential

452 expression findings could not be explained by previously described mechanisms (TPE ${ }^{26,27}$ and TPE-OLD $\left.{ }^{28}\right)$. This

453 suggests that there are other mechanisms by which telomere length regulates gene expression, or vice versa ${ }^{51}$. 
454 Follow-up experimental perturbation studies will be required to make this distinction, for example by inducing

455 telomere shortening (replicative senescence) followed by gene expression analysis or by inducing gene knockdowns

456 followed by telomere length measurements.

457 Finally, by making use of longitudinal data on participant survival, we were able to replicate a negative association

458 between telomere length and all-cause mortality ${ }^{96}$, although the small number of deceased participants (11) hampers

459 the wider interpretation of these results. Further studies of Lifelines participants will clarify this predicted

460 relationship ${ }^{97}$.

\section{Conclusions}

463 Telomere length is a complex trait in human populations whose variability is influenced by genetics, lifestyle,

464 intrinsic and environmental factors. Identification of such effects has been hampered by low reproducibility in

465 measurements, limited phenotypic information about study participants and lack of cellular resolution to study cell-

466 type-specific nuances. Applying reproducible methodology (Flow-FISH), we show that there is substantial variation

467 in telomere length among blood cell types that are usually measured together. We find a consistently lower telomere

468 length in males and determine the influence of genetics, intrinsic phenotypes and parental phenotypes and habits. In

469 particular, our identification of a novel effect of parental smoking habits highlights the importance of accounting for

470 common environmental influences usually overlooked in family-based telomere length heritability estimates.

471 Additionally, we propose a possible epigenetic mediation of maternal age effects on offspring telomere length. We

472 also linked telomere length variability with transcriptomic differences at the single-cell level. These results suggest

473 that T-cells are sensitive to telomere length-induced expression changes that can act through both short- and longer-

474 range interactions, but also that cell expression may directly influence telomere dynamics. Through gene expression

475 regulation, telomere shortening may contribute to ageing and age-associated diseases long before critical DNA

476 damage starts. Finally, we confirm telomere length as an independent risk factor for death, which emphasises the

477 importance of understanding the source of its variability in order to develop healthier life habits.

479 Acknowledgments

480 We thank J. Dekens for management, A. Maatman and M. Platteel for technical support and K. Mc Intyre for 481 English editing. This project was funded by the BBMRI grant for measuring telomere length and by a Rosalind 
482 Franklin Fellowship to A. Zhernakova. The researchers participated in this project are supported by Netherlands

483 Heart Foundation (IN-CONTROL CVON grants 2012-03 and 2018-27); the Netherlands Organization for Scientific

484 Research (NWO) Gravitation Netherlands Organ-on-Chip Initiative to J.F. and C.W.; NWO Gravitation Exposome-

485 NL (024.004.017) to J.F., A.K. and A.Z.; NWO-VIDI (864.13.013) and NWO-VICI (VI.C.202.022) to J.F.; NWO-

486 VIDI (016.178.056) to A.Z.; NWO-VIDI (917.14.374) to L.F.; NWO-VENI (194.006) to D.V.Z.; NWO-VENI

487 (192.029) to M.W.; NWO Spinoza Prize SPI 92-266 to C.W.; the European Research Council (ERC) (FP7/2007-

488 2013/ERC Advanced Grant 2012-322698) to C.W.; ERC Starting grant 637640 to L.F.; ERC Starting Grant 715772

489 to A.Z.; ERC Consolidator Grant (grant agreement No. 101001678) to J.F.; and RuG Investment Agenda Grant

490 Personalized Health to C.W. MM work is supported by RYC- 2017-22249 and PID2019-107937GA-I00 grants. T.S.

491 holds scholarships from the Junior Scientific Masterclass, University of Groningen[grant no. 17-34]. AR is funded

492 by a Formación Personal Investigador (FPI) grant [grant no. PRE2019-090193].

493 The Lifelines Biobank initiative has been made possible by a subsidy from the Dutch Ministry of Health, Welfare

494 and Sport; the Dutch Ministry of Economic Affairs; the University Medical Center Groningen (UMCG, the

495 Netherlands); the University of Groningen and the Northern Provinces of the Netherlands. The authors wish to

496 acknowledge the services of the Lifelines Cohort Study, the contributing research centres delivering data to Lifelines

497 and all the study participants.

\section{Material \& Methods}

\section{1. Lifelines Deep cohort}

$501 \quad$ Phenotype data description

502 Lifelines is a multi-disciplinary prospective population-based cohort study examining, in a unique three-generation

503 design, the health and health-related behaviours of 167,729 persons living in the North of the Netherlands. It

504 employs a broad range of investigative procedures to assess the biomedical, socio-demographic, behavioural,

505 physical and psychological factors that contribute to the health and disease of the general population, with a special

506 focus on multi-morbidity and complex genetics ${ }^{41}$. We collected data from the subcohort Lifelines DEEP $($ LLD, $\mathrm{n}=$

$5071,057,57.6 \%$ female, mean age (including months) 43.9 years [range 18-81.4 years]) $)^{32}$. Extensive information on

508 demographics, health and lifestyle factors including smoking and diet was collected via detailed questionnaires.

509 Mean BMI of participants was 25.1 [range 15.8-44.9]. Common age-related diseases within the cohort included 
510 hypertension (23\% of 841 participants with information), type 2 diabetes (1.3\% of 1,039 participants with

511 information) and hypercholesterolemia (14\% of 900 participants with information). In the cohort, $20 \%$ of

512 individuals smoked currently, $48 \%$ smoked for at least 1 year and $37 \%$ had mothers and $65 \%$ had fathers who

513 smoked.

514

515 Omics data description

516 Genome-wide genotyping data was generated as described in $^{32}$. Genotype data processing was done as described

$517 \mathrm{in}^{98}$. Briefly, microarray data were generated on CytoSNP and ImmunoSNP and then processed on the Michigan

518 Imputation Server ${ }^{99}$ to perform phasing using SHAPEIT and imputation using HRC version R1 as reference ${ }^{100}$. We

519 excluded SNPs with imputation quality $\mathrm{r}^{2}<0.5$, minor allele frequency (MAF) $<0.05$, call rate $<0.95$ and Hardy-

520 Weinberg equilibrium test $\mathrm{P}<1 \times 10^{-6}$, which resulted in 5,327,634 SNPs used in subsequent analyses. Genotype data

521 was available for 911 samples with non-missing telomere measurements.

522 Genome-wide methylation data generation for this cohort was described previously ${ }^{101}$. Briefly, the EZ DNA

523 Methylation kit (Zymo Research) was used to bisulfite-modify 500ng of genomic DNA, which was hybridised on

524 Illumina 450K array. Methylation probes were remapped to ensure their correct genomic location, and probes with

525 known SNPs at the single base extension site or CpG site were removed. Next, data was processed using a pipeline

526 described by Tost and Touleimat ${ }^{102}$. We used DASEN-normalised data with subsequent quantile normalisation and

527 probe scaling applied. Methylation data for 418,499 probes was available for 651 samples with telomere

528 measurements in at least one cell type.

529 2. Flow-FISH Telomere length measurement.

530 Telomere length measurements using automated multicolour flow-fluorescence in situ hybridisation (Flow-FISH)

531 was performed as described ${ }^{33}$. Briefly, white blood cells were isolated by osmotic lysis of erythrocytes in whole

532 blood using $\mathrm{NH}_{4} \mathrm{Cl}$. White blood cells were then mixed with bovine thymocytes of known telomere length (which

533 served as an internal control), denatured in formamide at $87^{\circ} \mathrm{C}$, hybridised with a fluorescein-conjugated

534 (CCCTAA)3 peptide nucleic acid (PNA) probe specific for telomere repeats and counterstained with LDS751 DNA

535 dye. The fluorescence intensity in granulocytes, total lymphocytes and lymphocyte subsets defined by labelled

536 antibodies specific for CD20, CD45RA and CD57 relative to internal control cells and unstained controls was

537 measured by flow cytometry to calculate the median telomere length from duplicate measurements. 
538 Out of a total 1,388 participants, we could not measure the telomere length in any cell type in 207. In addition,

539 granulocytes could not be measured in 109 participants, B-cells could not be measured in nine participants and NK-

540 cells could not be measured in 17 participants (all of these were different participants). We decided to remove all

541 participants with at least one missing cell type, which resulted in a final subset of 1,046 participants.

544 DNA from whole blood was isolated using the conventional protocol with Proteinase K digestion followed by

545 phenol extraction and isopropanol precipitation. Next, we performed a TaqMan quantitative real-time PCR approach

546 to quantify sjTREC expression (signal joint excision circles produced during T-cell development) using ViiA ${ }^{\mathrm{TM}} 7$

547 Real-Time PCR System (Life technologies). TaqMan qPCR was performed on 75-100 ng DNA in a $12 \mu$ reaction

548 mixture containing $18 \mu \mathrm{M}$ of each primer for sjTREC (5'-TCGTGAGAACGGTGAATGAAG-3' and 5'-

549 CCATGCTGACACCTCTGGTT-3') and for albumin as housekeeping gene (5'-TGAACAGGCGACCATGCTT-3'

550 and 5'-CTCTCCTTCTCAGAAAGTGTGCATAT-3') and $5 \mu \mathrm{M}$ of hydrolysis sjTREC probe 5'-(FAM)

551 CACGGTGATGCATAGGCACCTGC-3' (TAMRA) and albumin probe 5'-(FAM)

552 TGCTGAAACATTCACCTTCCATGCAGA -3' (TAMRA). PCR runs started with incubation at $50^{\circ} \mathrm{C}$ for 2 min,

553 then at $95^{\circ} \mathrm{C}$ for $15 \mathrm{~min}$, followed by 45 cycles of denaturation at $95^{\circ} \mathrm{C}$ for 15 sec and annealing/elongation at $60^{\circ} \mathrm{C}$

554 for $30 \mathrm{sec}$. All reactions were carried out in duplicate per sample using sjTREC primers and sjTREC probe as well

555 as albumin as a single-copy albumin gene to normalise the results, taking into account the amount of input DNA.

556 During PCR, the amplification mediated the cleavage of the probes, which contain a quencher (TAMRA) and a

557 reporter (FAM) dye. This, in turn, leads to the separation of the quencher from the reporter, thereby inducing

558 fluorescence of the reporter dye. The expression of target (sjTREC and albumin) in analysed samples was

559 established by measuring the threshold cycle (CT), defined as the cycle number at which the fluorescence generated

560 by cleavage of the probe passes a fixed threshold above baseline. We calculated the standard deviation between the

561 duplicates, and the results were accepted for further analysis when the standard deviation was $\leq 1$. Next, we

562 considered the average for all the duplicates with standard deviation $\leq 1$, and the normalised sjTREC expression in

563 each sample was calculated as a difference between average CT values of albumin and average sjTREC CT values.

\section{4. Definition of telomere fast and slow agers and comparison between biological ageing methods}


Telomere lengths were used to identify participants with above or below average telomere length. To do so, we

fitted a linear model to each cell type's telomere length using age, including months, as regressor. We then identified

567 participants under one standard deviation of their predicted value. We considered all participants with at least two

568 cell types passing the 1 standard deviation threshold to be fast or slow agers. Participants identified with some cell

569 types above 1 standard deviation and some below were excluded. Thus, we defined two groups of participants: fast

570 and the slow agers. We then performed a logistic regression using either sjTREC or methylation age as a regressor

571 explaining this binary category (fast agers or slow agers) while controlling for age, sex and cell counts.

\section{5. GREML heritability estimation}

573 We used the $\mathrm{GCTA}^{40}$ software for narrow sense heritability estimation. We used the microarray SNP data available

574 in LLD to calculate a genetic relationship matrix (GRM) using variants with a MAF > 1\% (GCTA --make-grm).

575 Next, we used the estimated GRM to calculate the amount of variance explained by the random effect of genetics in 576 a linear mixed model (GCTA --reml) while accounting for sex and age as fixed-effect covariates.

\section{6. GWAS of telomere length}

578 Using a previously described pipeline ${ }^{103}$, we performed an association analysis of telomere length of each cell type

579 with genome-wide SNP genotype data by calculating the Spearman correlation coefficient between telomere length

580 measurements corrected for age and sex and SNP genotype dosages. We corrected the results for multiple testing by

581 permuting genotype labels 10 times to create a null distribution that was used to control FDR at 0.05.

\section{7. Polygenic risk score calculation}

583 The contribution of genetics was calculated using a PRS created using 20 independent genome-wide significant loci

584 reported a large European-descent telomere GWAS study ${ }^{15}$. A weighted PRS was calculated for each LLD

585 participant using PLINK 1.9 --score sum function ${ }^{104}$. The PRS was used as a regressor using chronological age as

586 covariate and telomere length of each cell type as dependent variables of six different linear models.

\section{8. Phenotype correlations}

588 We preselected 90 phenotypes for correlation with telomere length based on the relevance and sample size of each

589 phenotype. We used telomere length as the dependent variable while the phenotype was standardised and used as the

590 explanatory variable. Linear models were fitted in R (v4.0.1) by ordinary least squares (OLS). We fitted all models

591 while accounting for the effect of age and sex. We then fitted a second model that also accounted for the effect of 
592 different blood cell counts available per sample, namely granulocytes (basophils, eosinophils and

593 neutrophils), erythrocytes, lymphocytes, monocytes and thrombocytes measured per plasma litre. We computed

594 FDR from all P-values using the Benjamini-Hochberg procedure ${ }^{105}$ as implemented in R base functions.

595 For some specific diabetes-related and BMI-related phenotypes, such as "Waist circumference in cm" and "Body

596 Mass Index $\left(\mathrm{kg} / \mathrm{M}^{2}\right)$ ", we included paternal and maternal age and parental smoking habits as additional covariates in

597 the model discussed above. At the same time, to disentangle maternal and paternal age effects, we applied a linear

598 model including covariates (age, sex, cell counts) and both phenotypes, using each telomere length as dependent

599 variable. We included an 11 penalisation (Ridge regression) using glmnet in $\mathrm{R}^{106}$ to shrink the estimates of

600 superfluous covariates. The strength of the regularisation parameter $(\lambda)$ was estimated by a 10-fold cross-validation.

601 To identify possible confounders in BMI-related phenotypes, we performed correlations between BMI and parental

602 smoking and parental age using an additional 10,000 participants from the Lifelines study population ${ }^{41}$.

\section{9. Assessment of total variance explained by phenotypes}

604 To assess the total variability explained by each layer of information, we selected all phenotypes found significantly

605 associated with at least two telomere lengths under an FDR of 0.05. We then fitted five nested models. First, we

606 regressed out the effect of chronological age in telomere length. Next, we fitted three nested models with intrinsic

607 host factors: one adding sex, a second adding cell counts and a third adding waist circumference and BMI. These

608 were fitted using a lasso shrinkage model (using glmnet in $\mathrm{R}^{106}$ ) to explain the residuals of telomere length after

609 removing the chronological age effect. The fourth model added the parental phenotypes age and smoking to the

610 intrinsic factors model. The final model added the PRS (see Polygenic Risk Score calculation) on top of the parental

611 model. We computed the variance explained $\left(\mathrm{R}^{2}\right)$ by each model and the $\Delta \mathrm{R}^{2}$ of the variability gained by each layer

612 of information introduced.

\section{10. Methylation association analyses}

614 We performed a GWAS of all probes with parental phenotypes using OLS in R. The following five phenotypes were 615 used: age of mother and father at participant's birth, maternal smoking during childhood, maternal smoking during

616 pregnancy and paternal smoking during childhood, all while correcting for age, sex and cell counts as covariates.

617 Bonferroni multiple testing correction was applied to correct for $5 x 418,499=2,092,495$ tests. Next, we assessed the 
618 association of methylation and telomere length using a similar linear model, adjusting for age, sex and cell counts as

619 covariates.

\section{11. Mediation analysis}

621 To test if the parental phenotype effect on telomere length may be mediated by methylation of certain probes, we

622 performed a mediation analysis. For this we selected triplets of parental phenotypes (independent variable),

623 methylation probes (mediator) and telomere length (dependent variable) using the following criteria: We required

624 that a parental phenotype was associated with the methylation probe with FDR $<0.05$, that methylation at this probe

625 was associated with telomere length at a nominal p-value threshold of 0.05 and that the parental phenotype was

626 associated with telomere length at a nominal p-value threshold of 0.05 . Next, we estimated mediation using the

627 mediation $\mathrm{R}$ package ${ }^{107}$, adding age, sex and cell counts as covariates to all models. We performed a Benjamini-

628 Hochberg multiple testing correction on the average causal mediation effect (ACME) p-value and required that the

629 FDR $<0.05$ and the average direct effect (ADE) p-value was $>0.05$ (meaning that the parental phenotype effect is

630 mostly mediated by methylation). In addition, we investigated a slightly different situation where methylation and

631 telomere length are swapped. Here we checked if the parental phenotype effect on methylation is mediated by

632 telomere length. While the real causality directions are very complex and often contain loops, and as a consequence

633 these swapped ACME P-values are often $<0.05$, we required that a swapped ACME p-value be larger than the main

634 original ACME p-value and that the mediated proportion of the effect for the original direction be larger than for the

635 swapped scenario.

\section{Differential expression analysis at the single-cell level}

scRNA-seq dataset features: library preparation, sequencing, alignment, pre-processing and quality control

To study gene expression changes with telomere length at the single-cell level, we used a subset of previously

642 telomere length data for at least one cell type in the current study. This scRNA-seq data was generated 5 years after

643 collection of the Flow-FISH telomere length data. In short, scRNA-seq data was generated using the 10X Chromium

644 Single Cell 3' V2 chemistry and libraries were 150bp PE sequenced on the NovaSeq6000. The Cellranger v3.0.2

645 pipeline was used with default parameters to demultiplex, generate FASTQ files, map reads to the GRCh37

646 reference genome and generate a unique molecular identifier (UMI)-corrected count matrix per cell ${ }^{108}$. After quality

647 control, 54,373 cells remained. In the original dataset, k-nearest neighbour clustering was used to cluster the cells. 
648 We then performed automated cell type classification using Azimuth to annotate the cells ${ }^{50}$. In detail, we conducted

649 a supervised analysis guided by a reference dataset to enumerate cell types that would be challenging to define with

650 an unsupervised approach. Thus, we mapped our scRNA-seq query dataset to a recently published CITE-seq

651 reference dataset of 162,000 PBMC measured with 228 antibodies $^{50}$. For this process, we used a set of specific

652 functions from the Seurat R package v4.0.0 $0^{50,109}$. First, we normalised the reference dataset using the SCTransform

653 function. Then, we found anchors between reference and query using a precomputed supervised PCA transformation

654 through the FindTransferAnchors function. Afterwards, we transferred cell type labels and protein data from the

655 reference to the query. We also projected the query data onto the UMAP structure of the reference. For these two

656 last steps, we used the FindTransferAnchors function. Finally, the high resolution cell-type-annotations predicted by

657 Azimuth (celltype.12) were combined in such a way to more closely reflect the resolution of the Flow-FISH

658 annotations (i.e. naïve and memory CD4T and CD8T cells, NK- and B-cells) (Supplementary Table 6).

659 Differential expression analysis at the single-cell level

660 Telomere length DE analyses were conducted using MAST v1.16.0 $0^{110}$ at single-cell resolution (sc-DEA) by

661 selecting the matched telomere length measurement and gene expression level for each of these cell types. As a first

662 approach (I), we performed an independent telomere length sc-DEA for each of the previously defined cell types

663 (i.e. naïve and memory CD4T and CD8T cells, NK- and B-cells). We then conducted a second approach (II) in we

664 combined multiple (sub)cell types together in the same analysis (i.e. we performed five different sc-DEA

665 combining: all CD4T, CD8T, naïve T, or memory T and T-cells) while controlling for cell type annotation

666 (Supplementary Table 6). As our first approach had indicated that we had insufficient cells to detect telomere

667 length-associated DE genes in B- and NK-cells, we did not re-analyse these cells using our second approach. This

668 strategy allowed us to increase the number of cells per donor per analysis and thereby increase the power to detect

669 potential effects. In both cases, as input, we used the log-normalised and scaled expression counts from those genes

670 expressed in at least 10\% of the cells. MAST uses a two-part generalised linear model, specifically a Hurdle model,

671 on zero-inflated continuous data in which the zero process is modelled as a logistic regression and the continuous

672 process is modelled as a linear regression. To accommodate the complex experimental design while controlling for

673 covariates, including both biological variables (sex, age, donor and cell type) and technical factors (cellular

674 detection rate (CDR) and 10x lane/experimental batch), we fitted a general linear mixed model (glmer) of the form: 


$$
\text { Expr }_{\mathrm{i}} \sim \text { telomere length } \mathrm{i}_{\mathrm{i}}+\mathrm{CDR}_{\mathrm{i}}+\text { sex }+ \text { age }+ \text { celltype. } 12_{\mathrm{i}}+(1 \mid \text { donor })+(1 \mid \text { lane) (II) }
$$

678 where Expr $_{\mathrm{i}}$ is the log-normalised, scaled expression of the gene being tested in cell $\mathrm{i}$; telomere length $\mathrm{h}_{\mathrm{i}}$ is the 679 telomere length measurement using Flow-FISH in the tested cell type; $\mathrm{CDR}_{\mathrm{i}}$ is the fraction of genes that are 680 detectably expressed in each cell; celltype. $12_{\mathrm{i}}$ is the high resolution cell type annotation predicted by Azimuth; sex 681 and age are phenotypic variables from the donors; donor is the donor LLD id and lane is the experimental batch, 682 here being the lane of a 10X chip. First, we used the zlm function to fit a glmer (method = 'glmer', ebayes=FALSE, 683 fitArgsD=list, $\mathrm{nAGQ}=0$, all other parameters set to default). Some of the gene models failed to converge when 684 considering random effects (i.e. donor and lane), which resulted in NA estimated coefficients. To mitigate this 685 convergence issue, $\mathrm{nAGQ}=0$ was passed to the fitting function. We then used the summary function to perform a 686 likelihood ratio test (LRT) to test for differences when we drop the telomere length factor (doLRT=telomere length, 687 fitArgsD=list, $\mathrm{nAGQ}=0$, all other parameters set to default).

688 Finally, genes were considered to be DE with telomere length when gene expression change was significant at an 689 FDR $<0.05$. The EnhancedVolcano function from EnhancedVolcano R package v1.8.0 was used to visualise the 690 gene's significance and $\log 2 \mathrm{FC}$. The pheatmap function from pheatmap R package v1.0.12 was used to visualise the 691 expression pattern (log-normalised counts), clustering and annotation of the set of 97 unique DE among T-cells. For 692 visualisation purposes, we down-sampled each of the Azimuth's predicted T (sub) cell types to 100. To distinguish 693 the four main expression patterns, the argument cutree_rows $=4$ was set when using the pheatmap function. The 694 pheatmap function was also used to visualise the LFC per telomere length unit among the set of 97 unique DE genes 695 identified in T, CD4T and CD8T cells.

Literature analysis

697 To validate our sc-DEA findings, we compared our results to four biologically related studies that: i) revealed novel 698 telomere proteins using in vivo cross-linking, tandem affinity purification and label-free quantitative LC-FTICR-

$699 \mathrm{MS}^{51}$, ii) identified a set of genes associated with ageing in whole blood using bulk RNA-seq after correcting for cell 700 type composition ${ }^{53}$, iii) created the GenAge human database as part of the HAGR databases composed of 307 701 human ageing-related genes ${ }^{54}$ and iv) defined an association between telomere length and methylated cytosine 
702 levels for both blood and EBV-transformed cell-line DNA samples ${ }^{29}$. To this end, we overlapped the set of 97

703 unique genes we found to be DE with telomere length against these four previously reported lists of genes and

704 performed a two-sided Fisher's exact test to assess whether these overlaps were significant. We could not test the

705 overlap with ${ }^{29}$ since multiple methylated $\mathrm{CpG}$ sites are linked to the same gene. For the tested overlaps, as our

706 background list of genes, we gather the union of all tested genes in T-, CD4T and CD8T cells. As each study's

707 background list of genes, we considered all the tested genes in ${ }^{53}$ and all the protein-coding genes (using Gencode

708 v26, GRCh38 annotation) in ${ }^{51}$ and ${ }^{54}$.

709 Functional enrichment analysis

710 We performed a functional enrichment analysis through an over-representation analysis using WebGestalt (WEB-

711 based GEne SeT AnaLysis Toolkit) ${ }^{111}$. As the input gene list, we used the 44 and $91 \mathrm{DE}$ genes we identified in

712 CD4T and all T-cells, respectively, split by their direction of effect (i.e. positively or negatively associated with

713 telomere length). As the background gene set, we used the 2,426 and 2,427 expressed genes that were tested in the

714 sc-DEA of the CD4T or all T-cells, respectively. As functional databases, we used two different pathway databases:

715 KEGG and Reactome. Advanced default parameters were used (minimum and maximum number of genes for a

716 category: 5 and 2000, multiple test adjustment: $\mathrm{BH}$, significance level: FDR $\leq 0.05$, number of categories expected

717 from set cover: 10, redundancy reduction: affinity propagation and weighted set cover).

718 Differentially expressed genes telomere distance analysis

719 We compared the shortest distance to the telomere of DE genes compared to non-DE genes in CD4T cells and T-

720 cells. Gene locations were retrieved using the human Ensembl BioMart dataset GRCh38.p13 ${ }^{112}$. Chromosome length

721 was used as a proxy for telomere location. We took the minimum between the start of the gene position and the

722 distance between the end of the gene and the end of the chromosome as the distance from the gene to the telomere.

723 We then performed a one-sided Fisher's exact test to examine whether there was an enrichment of genes located at

724 subtelomeric regions in the DE sets in comparison to background non-DE sets. We performed this analysis in

725 positively and negatively telomere-associated genes separately and in each of the two cell types. To overcome a

726 possible confounder effect of chromosome length, we subsampled with no replacement genes from the non-DE gene

727 set while keeping the same chromosome proportions observed in the DE gene set, and again performed a Fisher's

728 exact test. 


\section{Survival analysis}

730 We obtained survival information for 1,044 participants and ultimately the dates on which 11 participants had died.

731 We measured the number of survival days since blood was donated. The R 'survival' package was used to perform a

732 Cox regression analysis using survival (days) as dependent variable and telomere length as explanatory variable,

733 while controlling for age, sex and blood cell counts. We tested the proportional hazards assumption, both of

734 telomere length as a covariate and for the whole model (cox.zph function), using a p-value cut-off of 0.05 .

\section{Supplementary Materials}

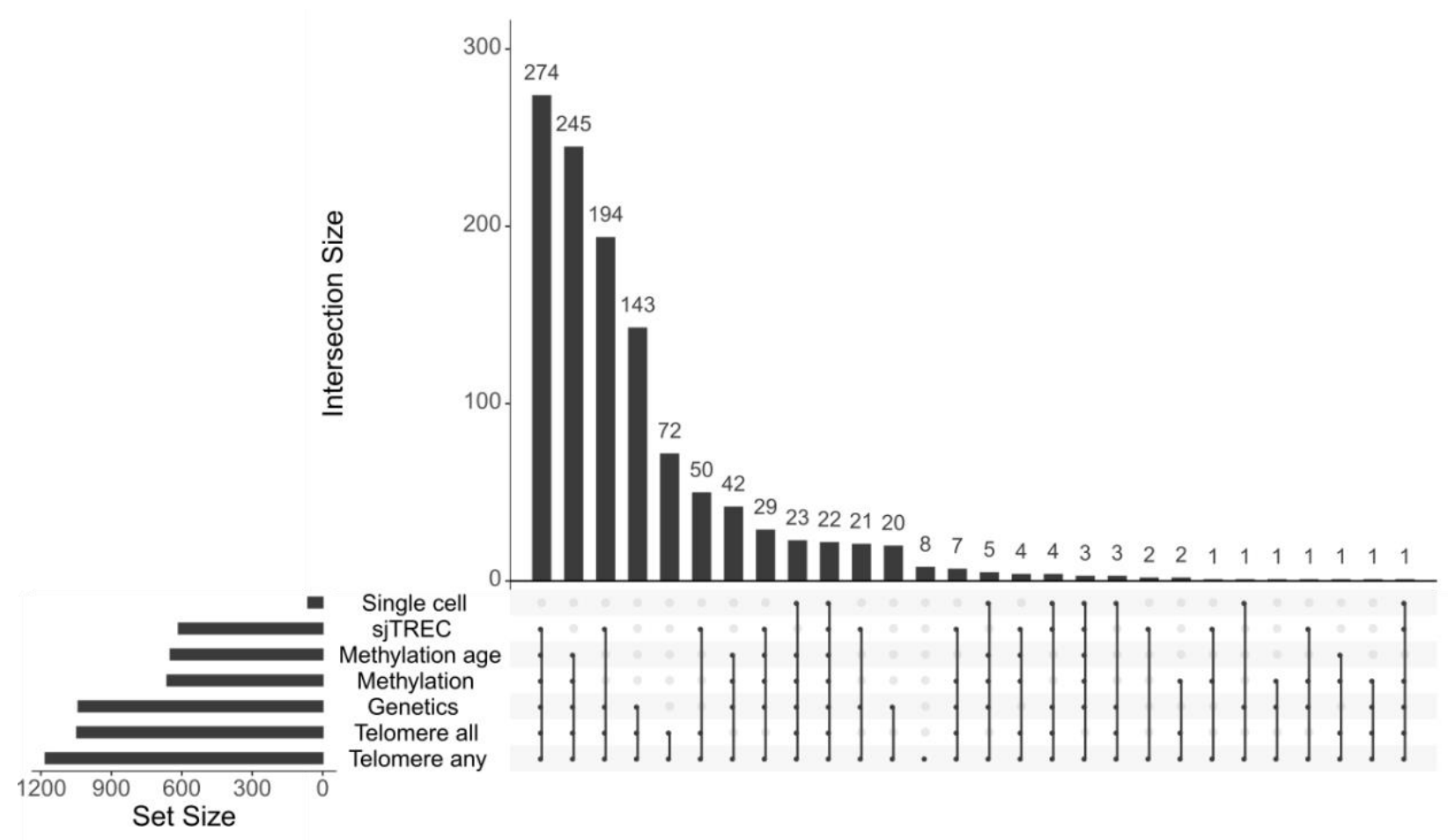

739 Supplementary Figure 1. Phenotype markers in the LLD cohort. Representation of omics data used in this work.

740 Horizontal bars indicate the number of Lifelines participants who had available information for the specific omics

741 layer. Vertical lines indicate the number of samples in the intersection of the different layers and not seen in any

742 other layer. Single cell (62) indicates the number of samples with single-cell RNA-seq data. sjTREC (613) indicates

743 the number of people with qPCR measurements of sjTRECs. Methylation age (648) is a subset of participants where

744 age was predicting using the methylation probes. Methylation (662) indicates the number of participants with

745 available methylation arrays. Genetics $(1,040)$ is the number of participants with available microarray data. 
746 Telomere any $(1,180)$ are the number of participants with at least one cell population where telomere length was

747 measured. Telomere all $(1,046)$ are the number of participants with telomere lengths for all six cell populations

748 available.

749 
bioRxiv preprint doi: https://doi.org/10.1101/2021.12.14.472541; this version posted December 15,2021 . The copyright holder for this preprint (which was not certified by peer review) is the author/funder. All rights reserved. No reuse allowed without permission.

A
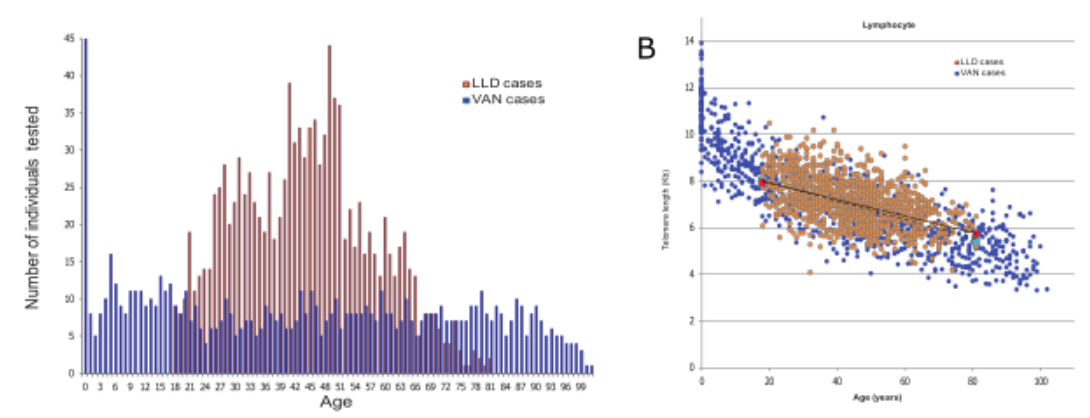

c
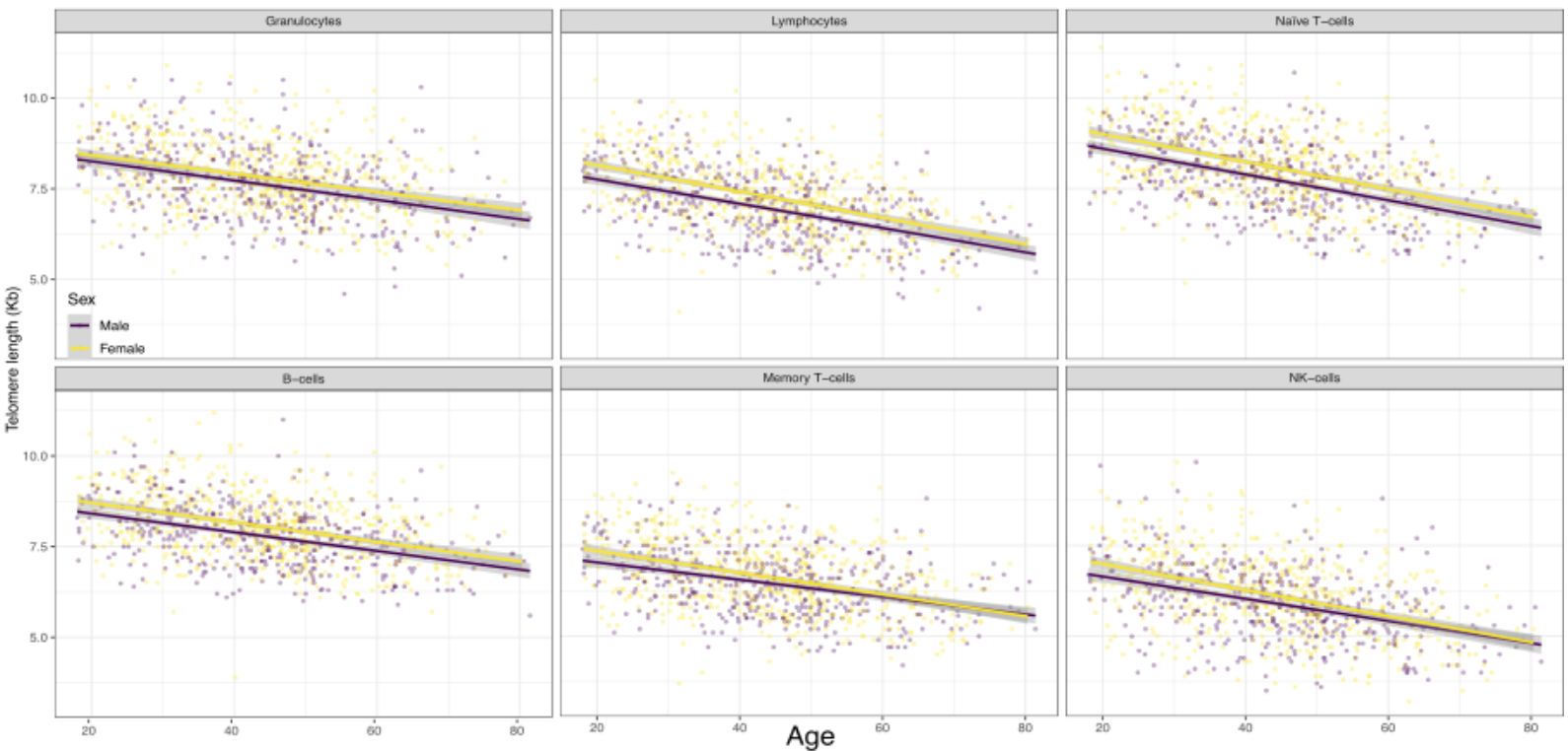

D

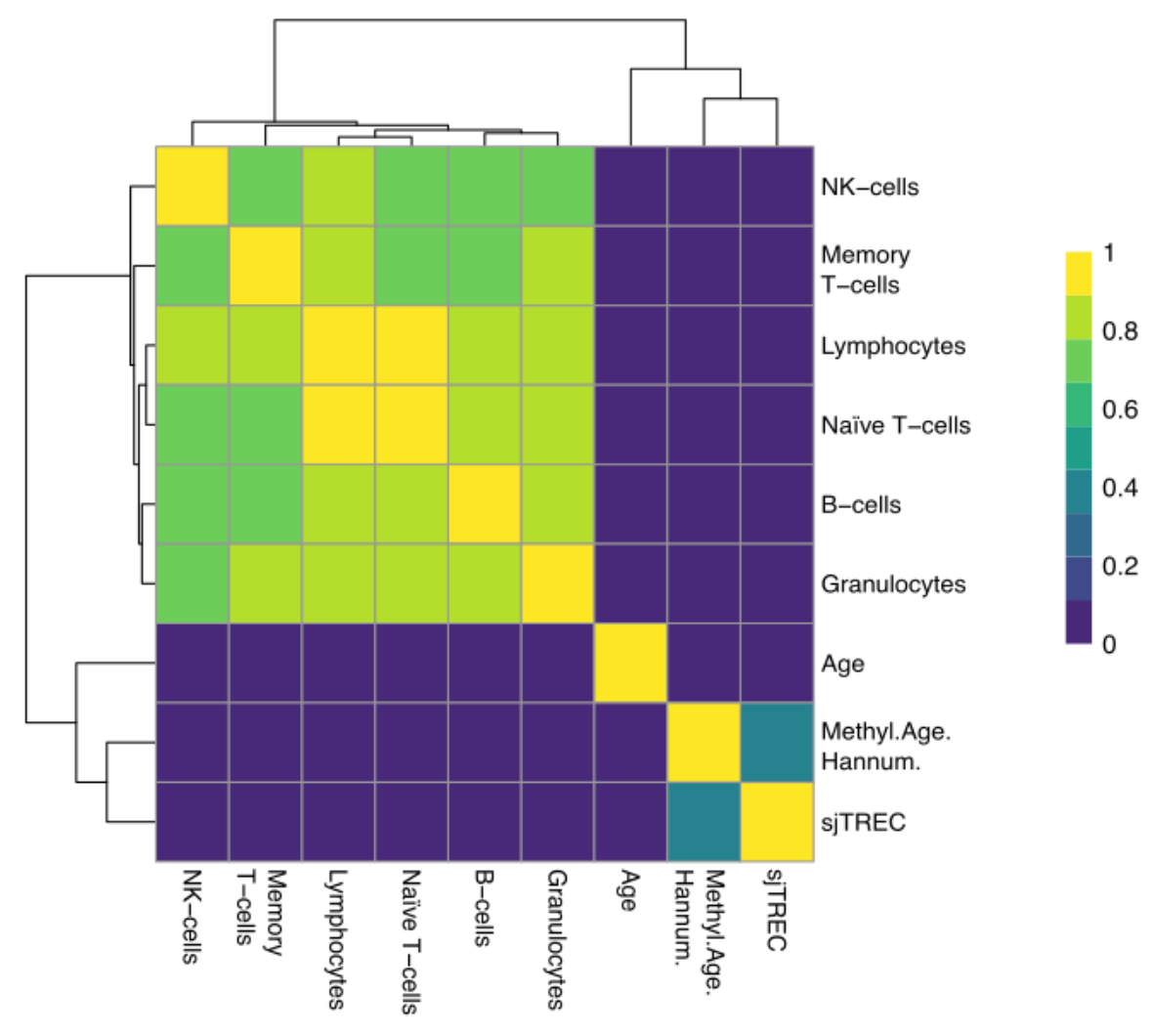


751 Supplementary Figure 2. Sample cohort descriptions and measurements comparison. A. Number of study

752 participants per age year in the Canadian study (VAN, blue, total $\mathrm{n}=835$, ages $18-81, \mathrm{n}=490$ ) and the LLD study

753 (LLD, orange, total $\mathrm{n}=1049)$. B. Total lymphocyte cell type telomere length measurements $(\mathrm{Kb})$ comparison

754 between studies. Canadian cohort in blue. LLD cohort in orange. Each point represents an individual. $50^{\text {th }}$

755 percentiles of distribution are shown for each cohort and are consistent with one another (Canadian cohort light blue

756 boundaries and dashed line, LLD cohort red boundaries and full line). The higher variability between models at

757 older age is attributed to differences in study design and recruitment. C. Telomere length decreases with age in six

758 different cell types. Each point represents a participant's telomere length $(\mathrm{Kb})$ in each cell type. Colour shows the

759 sex of the participant. Trend line presents the average telomere length for a given age and sex group. Grey area

760 surrounding trend lines indicates the 95\% confidence interval of the trend line. D. Correlation (Spearman's rho

761 value) between the absolute value of telomere lengths, Hannum-based methylation age and sjTREC qPCR relative

762 expression residuals after chronological age (Age) regression.
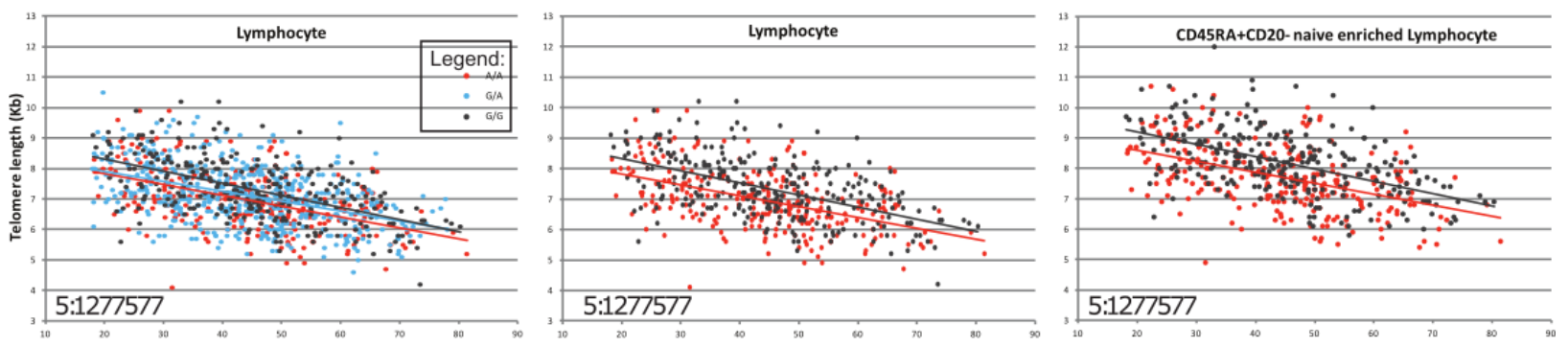

Supplementary Figure 3. Telomerase SNP genotype with significantly different telomere length. TERT rs33961405 (chromosome 5:1277577) genotypes and respective linear regression are shown for the lymphocyte cell subset, with homozygous genotypes displayed for the lymphocyte and the CD45RA+CD20-naïve enriched cell type. 


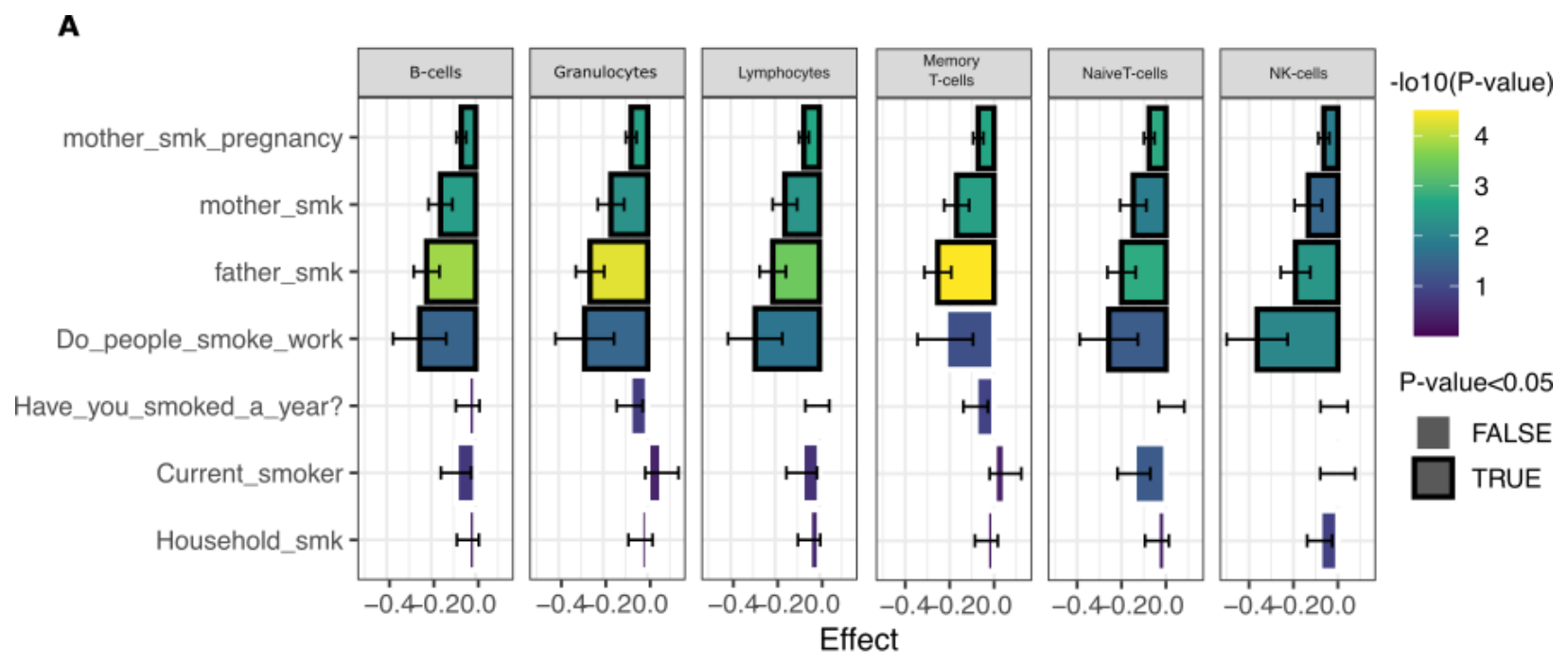

\section{B}

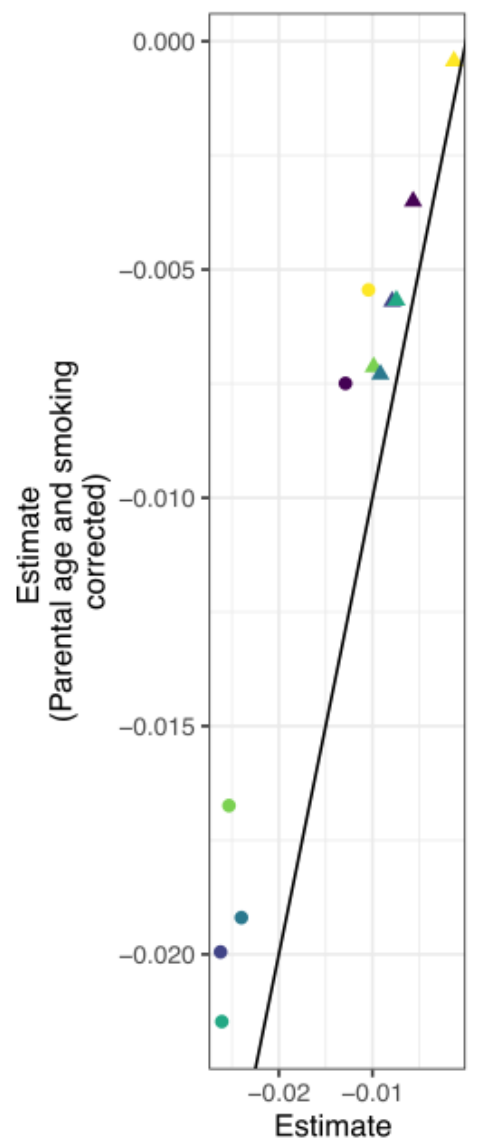

(Parental age and smoking uncorrected)

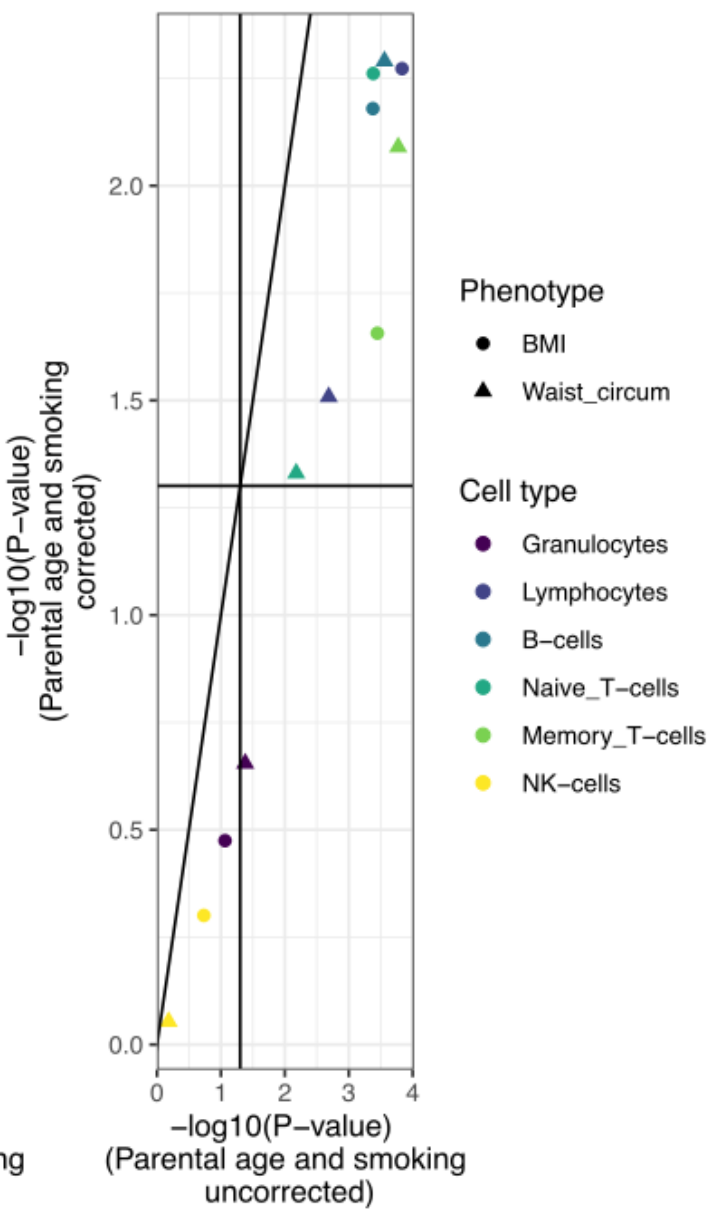

Supplementary Figure 4. Phenotype exploration. A. Smoking effects on participant telomere length. Length of

774 bar represents the linear model estimate. Colour shows the estimated P-value. Black outline indicates significant 
associations (nominal $\mathrm{P}<0.05$ ). Error bars indicate the standard deviation of the estimation. Most phenotypes contain two levels (yes/no), except household_smk, which is a continuous variable of the number of people smoking at the

777 participant's residence, and mother_smk_pregnancy, which is a 4-level factor that includes: mother did not smoke,

778 mother smoked before pregnancy, mother quit/reduced smoking during pregnancy and mother kept smoking

779 regularly during pregnancy. B. BMI phenotypes corrected for parental age and smoking status. Left panel shows

780 estimated effect size of BMI (circles) and waist circumference (triangles) on telomere length, while controlling for

781 parental age and smoking status (y-axis) and without correction (x-axis). Associated -log10(P-values) of the

782 estimates are shown in the right panel. Vertical and horizontal lines represent the nominal P-value significance

783 threshold (0.05).

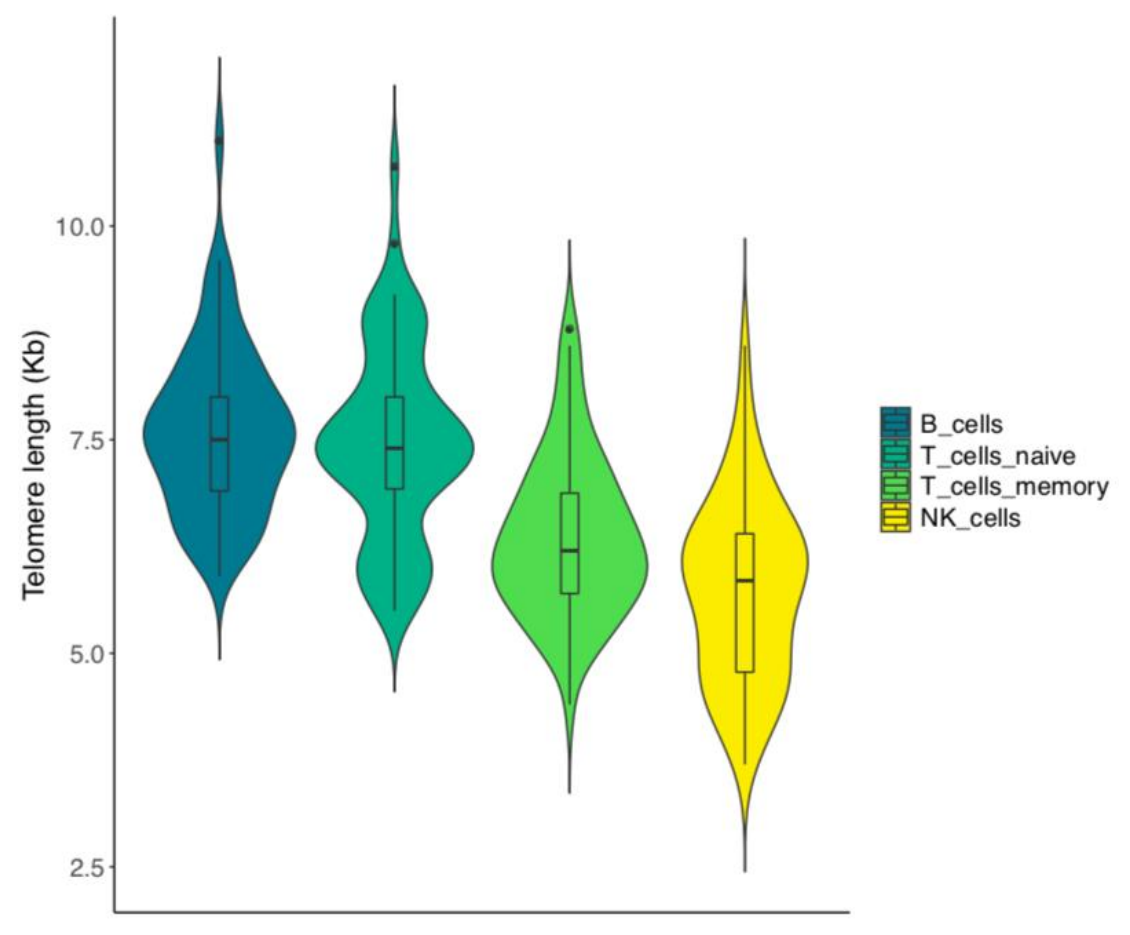

787 Supplementary Figure 5. Telomere length in the subpopulations with single-cell expression data. Distribution

788 of telomere length by cell type in the subset of 62 LLD donors for which both scRNA-seq and Flow-FISH telomere

789 length data was collected. We confirmed that this subset of 62 LLD donors showed similar telomere length

790 distributions to the total study population of 1,046 LLD donors in which telomere length was assessed (Figure 1A). 

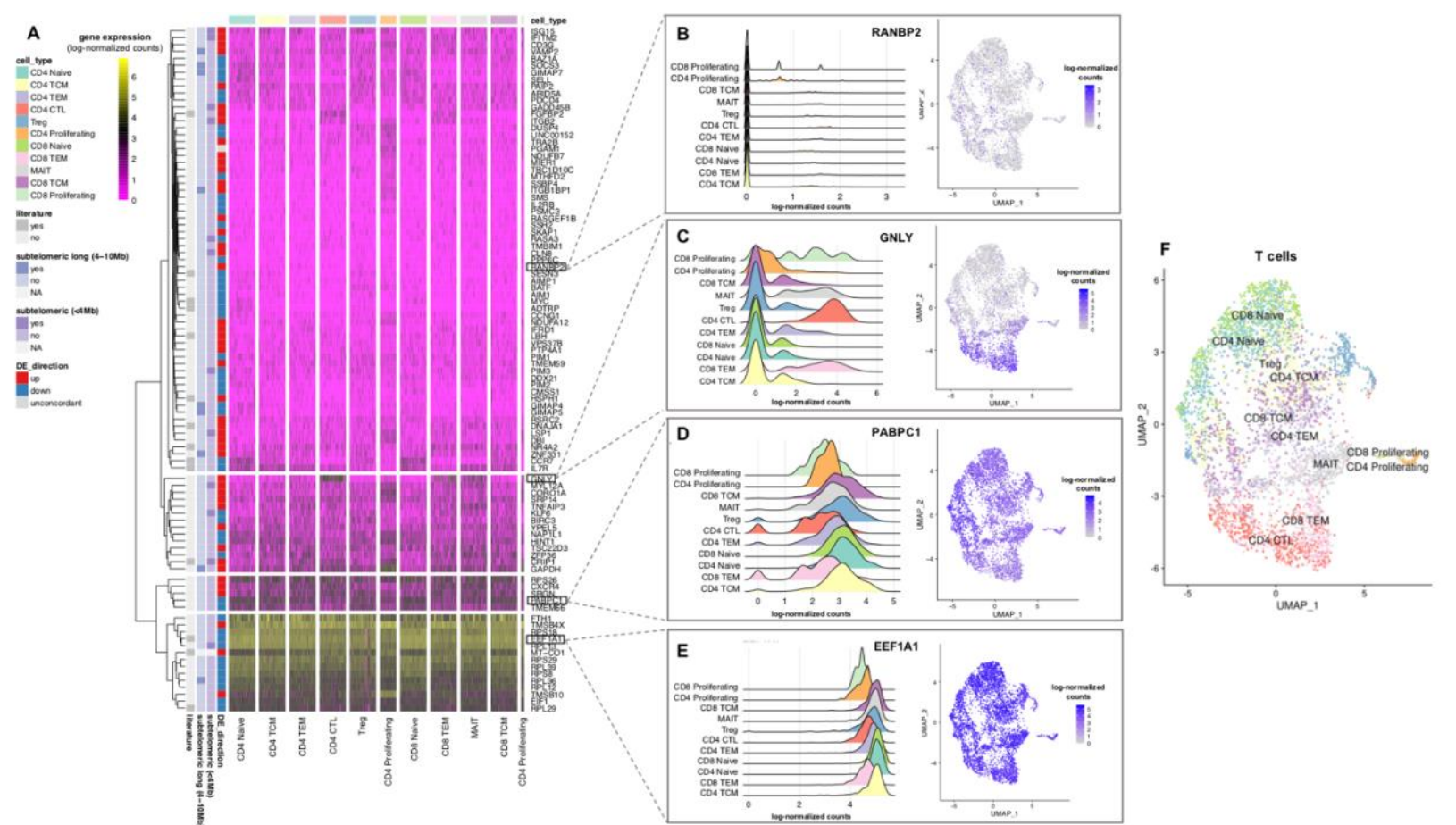

793 Supplementary Figure 6. Gene expression pattern of the DEGs obtained through the DEA approach II across

794 Azimuth's predicted T-(sub)cell types. A. Heatmap of the gene expression (log-normalised counts) pattern of the

795 set of 97 unique DEGs identified in T-, CD4T and CD8T cells. The hierarchical clustering of the genes revealed four

796 main expression patterns. From bottom to top: genes highly and ubiquitously expressed, genes moderately and

797 ubiquitously expressed, genes preferentially expressed in a particular cell type, and genes lowly and ubiquitously

798 expressed. The column annotation bar shows all the cells from the same cell type. The row annotation bars show the

799 DEG direction (DE_direction), whether the DEG is located at the subtelomeric (<4Mb) or subtelomeric long (4-

$80010 \mathrm{Mb}$ ) region, and if the DEG was previously reported in any of the following studies: Pellegrino-Coppola et al.,

$8012021^{53}$, Tacutu R et al., $2018^{54}$, Buxton JL et al., $2014^{29}$ or Nittis T et al., $2010^{51}$ (literature) (Supplementary Table

802 8). Only one DEG (PGAM1) showed a different direction among T, CD4T and CD8T DEAs (DE_direction =

803 unconcordant). The distance to the telomeres was not calculated for the mitochondrial gene MT-CO1 (subtelomeric

$804(<4 \mathrm{Mb})$ and subtelomeric long $(4-10 \mathrm{Mb})=\mathrm{NA})$. For visualisation purposes, we down-sampled each of the cell types

805 to 100 cells. B-E. Examples of the four main gene expression patterns described in (A). Left: Gene expression (log-

806 normalised counts) distribution plots across T (sub) cell types. Right: T-cells UMAP plots coloured by gene

807 expression (log-normalised counts). F. T-cells UMAP plot coloured by Azimuth's predicted T (sub) cell types. In B-

808 F, we down-sampled each of the cell types to 500 cells for visualisation purposes. 

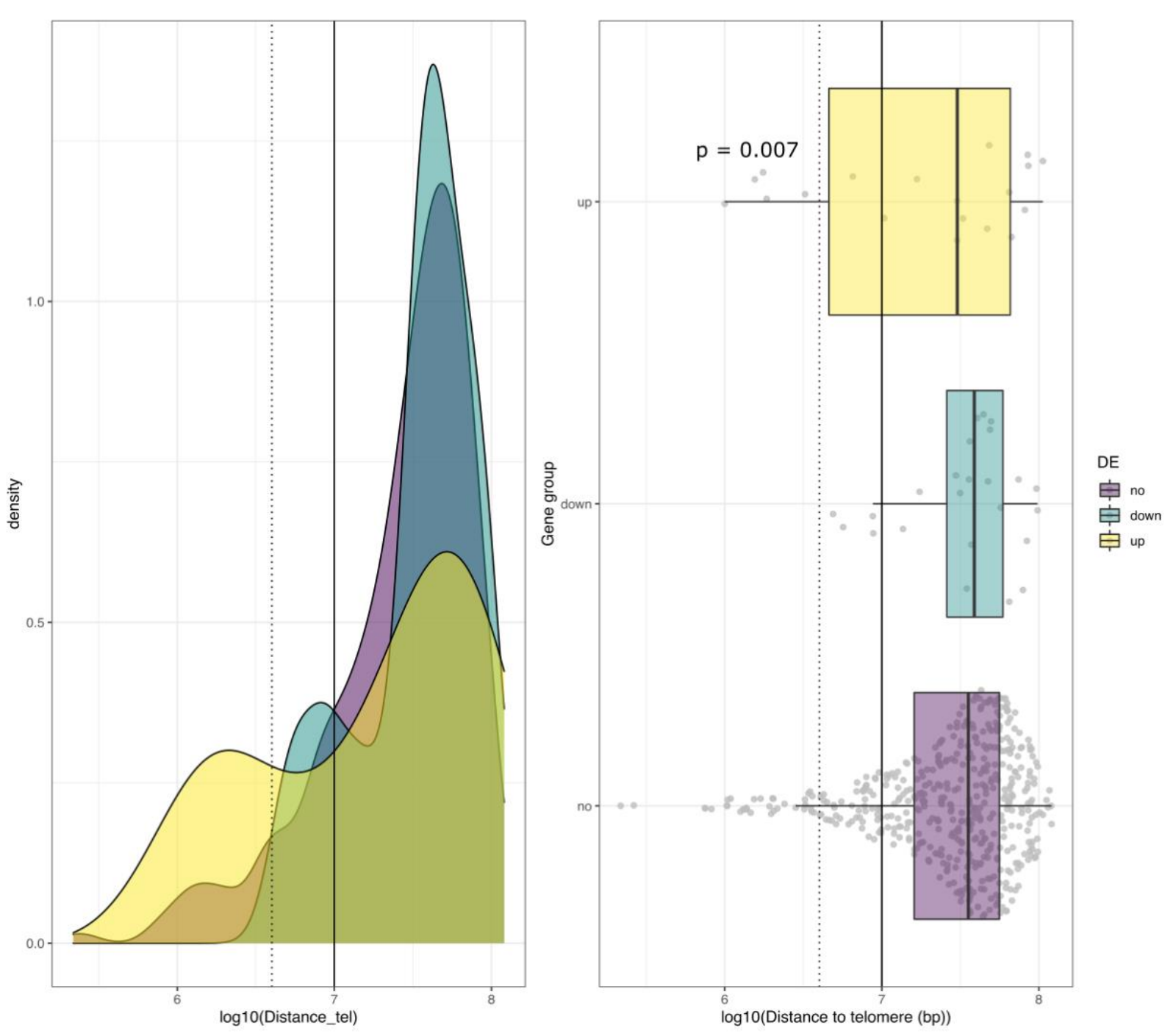

811 Supplementary Figure 7. Distance to telomere of DE gene sets. Comparison of distance to the telomeres between

812 DE and background genes in CD4 T-cells. A. Distribution of distance to telomere of DE genes (downregulated

813 (blue) and upregulated (yellow) ) and background (purple). B. Tukey boxplot of background genes, genes negatively

814 associated with telomere length and genes positively associated with telomeres. Vertical lines represent subtelomeric

815 region length (dotted, $4 \mathrm{Mb}$ ) and maximum distance where TPE-OLD has been detected (not dotted, 10Mb).

817 Supplementary Table 1. Descriptive statistics (mean age and BMI and female proportion) and number of

818 participants in each of the data layers used in the study. 
820 Supplementary Table 2. Telomere genome association summary statistics. Table 1.1 Replication of SNPs

821 reported in Li et al. ${ }^{15}$. Table 1.2 Summary statistics of associations which $\mathrm{p}<1 \times 10^{-5}$. EA: effect allele, beta:

822 estimated effect of effect allele load in telomere length, SE: standard error of the estimated effect.

824 Supplementary Table 3. Exploratory statistics of phenotypes used. NA: number of missing values. Not NA:

825 number of samples used for analysis of the phenotype. Info: Maximum, minimum, mean and standard deviation of

826 continuous phenotypes. For categorical phenotypes, it counts the number of participants per category.

828 Supplementary Table 4. Summary statistics of associations between telomere length and phenotypes. Table

829 4.1 Summary statistics of the model controlling for sex and gender. Table 4.2 Summary statistics of the model

830 controlling for sex, gender and blood cell composition. Regressor: phenotype which effect the summary statistics

831 makes reference to. Dependent: telomere length cell type used a dependent variable. $N$ : number of samples used for

832 assessment. Levels: number of levels (if categorical variable). Level_n: number of samples per level (if categorical)

833 or number of samples (continuous). FDR: false discovery rate estimated using Benjamini-Hochberg.

835 Supplementary Table 5. Methylation and mediation analysis summary statistics. Probe: methylation site

836 assessed; $e Q T M \_g e n e \_p$ 0.05: gene which expression is associated with methylation of this probe with a p-value <

$8370.05^{101}$; mtl: telomere length measurement; \{probe,pheno,mtl $\}-\{$ probe,pheno,mtl $\}$ : association estimated effect

838 between methylation (probe), phenotype (pheno) or telomere length(mt); fit.totaleffect: association p-value between

839 telomere length (outcome) and phenotype (predictor); fit.mediator: association p-value between methylation

840 (outcome) and phenotype (predictor), fit.dv: association p-value between telomere length (outcome) and phenotype

841 (predictor) when methylation is added as one of predictors; pcor: perarosn correlation, pheno = phenotype,

$842 \mathrm{mtl}=$ telomere length, probe=methylation site; $A C M E$ (average causal effects): pval (pvalue), BH(Benjamini-

843 Hochberg FDR), estimate (effect estimate); $A D E$ (average direct effects); Prop_med: proportion of the effect of

844 phenotype on telomere length that goes through methylation (mediator); ACME_pval_reverse: average causal

845 mediation effects p-value for the opposite direction (effect of phenotype on methylation is mediated by telomere

846 length); $A D E \_p v a l \_r e v e r s e$ : average direct effects p-value for the opposite direction (effect of phenotype on

847 methylation is mediated by telomere length); Prop_med_reverse: proportion of the effect of phenotype on 
methylation that goes through telomere length; pass_filter: Boolean value showing if the mediation result passes all

filters

851 Supplementary Table 6. Cell type classification. Relationship between the cell type classification used for the two differential expression analysis approaches (DE_approach_I and DE_approach_II) and the automated high resolution

853 (Azimuth_celltype_12) cell type annotation using Azimuth. The number of cells $(n)$ within each of the 854 Azimuth_celltype_I2 cell type-annotations is shown. The Azimuth_celltype_I2 cell types are sorted from high to low $n$, within each of the levels of the DE_approach_I cell type resolution.

Supplementary Table 7. Differential Expression Analysis (DEA) results. Summarised model features from a ZlmFit object using MAST. Table 7.1 DEA results for DE method 1. Table 7.2 DEA results for DE method 2. Primerid: the gene; coef: point estimate; ci.hi and ci.low: upper and lower bound of confidence interval, respectively; z: z score (coefficient divided by standard error of coefficient); $\operatorname{Pr}(>C h i s q)$ : likelihood ratio test p-value; fdr: False Discovery Rate, multiple testing correction method. Columns contain NAs if they are not applicable for a particular component or contrast.

Supplementary Table 8. Differentially Expressed Genes (DEGs) features. Summary information table for the unique DEGs (unique_DEGs) in each of the cell types (group) discovered using the DE_approach_II. For each unique DEG, we report information on their DE direction (DE_direction), whether the DEG is located at the subtelomeric $(<4 \mathrm{Mb})$ or subtelomeric long $(4-10 \mathrm{Mb})$ region and if the DEG was previously reported in any of the following studies: Coppola et al., 2021, Tacutu R et al., 2018, Buxton JL et al., 2014 or Nittis T et al., 2010 (literature).

Supplementary Table 9. Functional Enrichment Analysis (FEA) results. FEA results obtained through an overrepresentation analysis (ORA) by WebGestalt (WEB-based GEne SeT AnaLysis Toolkit) using the set of positively

872 or negatively associated (as specified in the DE_diretion column) DEGs with telomere length in CD4T and T-cells 873 (as specified in the cell_type column) by DEA approach II. 


\section{Bibliography}

878

879

880

881

882

883

884

885

886

887

888

889

890

891

892

893

894

895

896

897

898

899

900

1. Levy, M. Z., Allsopp, R. C., Futcher, A. B., Greider, C. W. \& Harley, C. B. Telomere endreplication problem and cell aging. J. Mol. Biol. 225, 951-960 (1992).

2. Müezzinler, A., Zaineddin, A. K. \& Brenner, H. A systematic review of leukocyte telomere length and age in adults. Ageing Res. Rev. 12, 509-519 (2013).

3. Wilson, V. L., Smith, R. A., Ma, S. \& Cutler, R. G. Genomic 5-methyldeoxycytidine decreases with age. J. Biol. Chem. 262, 9948-9951 (1987).

4. Jung, M. \& Pfeifer, G. P. Aging and DNA methylation. BMC Biol. 13, 7 (2015).

5. Steinmann, G. G. Changes in the Human Thymus During Aging. in The Human Thymus: Histophysiology and Pathology (ed. Müller-Hermelink, H. K.) 43-88 (Springer Berlin Heidelberg, 1986).

6. Evans, S. K., Bertuch, A. A. \& Lundblad, V. Telomeres and telomerase: at the end, it all comes together. Trends Cell Biol. 9, 329-331 (1999).

7. Blackburn, E. H. Structure and function of telomeres. Nature 350, 569-573 (1991).

8. Aubert, G. \& Lansdorp, P. M. Telomeres and aging. Physiol. Rev. 88, 557-579 (2008).

9. Aubert, G., Baerlocher, G. M., Vulto, I., Poon, S. S. \& Lansdorp, P. M. Collapse of telomere homeostasis in hematopoietic cells caused by heterozygous mutations in telomerase genes. PLoS Genet. 8, e1002696 (2012).

10. Lingner, J., Cooper, J. P. \& Cech, T. R. Telomerase and DNA end replication: no longer a lagging strand problem? Science 269, 1533-1534 (1995).

11. von Zglinicki, T. Oxidative stress shortens telomeres. Trends Biochem. Sci. 27, 339-344 (2002).

12. Codd, V. et al. Identification of seven loci affecting mean telomere length and their 
association with disease. Nat. Genet. 45, 422-7, 427e1-2 (2013).

902 13. Dorajoo, R. et al. Loci for human leukocyte telomere length in the Singaporean Chinese

903 population and trans-ethnic genetic studies. Nat. Commun. 10, 2491 (2019).

904 14. Mangino, M. et al. Genome-wide meta-analysis points to CTC1 and ZNF676 as genes

905 regulating telomere homeostasis in humans. Hum. Mol. Genet. 21, 5385-5394 (2012).

906 15. Li, C. et al. Genome-wide Association Analysis in Humans Links Nucleotide Metabolism to

907 Leukocyte Telomere Length. Am. J. Hum. Genet. 106, 389-404 (2020).

908 16. Taub, M. A. et al. Novel genetic determinants of telomere length from a trans-ethnic

909 analysis of 109,122 whole genome sequences in TOPMed. bioRxiv (2019)

$910 \quad$ doi:10.1101/749010.

911 17. Rufer, N. et al. Telomere fluorescence measurements in granulocytes and Tymphocyte

912 subsets point to a high turnover of hematopoietic stem cells and memory T cells in early

913 childhood. J. Exp. Med. 190, 157-167 (1999).

914 18. Dugdale, H. L. \& Richardson, D. S. Heritability of telomere variation: it is all about the

915 environment! Philos. Trans. R. Soc. Lond. B Biol. Sci. 373, (2018).

916 19. Broer, L. et al. Meta-analysis of telomere length in 19,713 subjects reveals high heritability,

917 stronger maternal inheritance and a paternal age effect. Eur. J. Hum. Genet. 21, 1163-1168

$918 \quad$ (2013).

919 20. Demanelis, K. et al. Determinants of telomere length across human tissues. Science 369,

$920 \quad$ (2020).

921 21. Gadalla, S. M., Cawthon, R., Giri, N., Alter, B. P. \& Savage, S. A. Telomere length in

922 blood, buccal cells, and fibroblasts from patients with inherited bone marrow failure

$923 \quad$ syndromes. Aging 2, 867-874 (2010). 
924 22. Aubert, G., Hills, M. \& Lansdorp, P. M. Telomere length measurement-caveats and a

925 critical assessment of the available technologies and tools. Mutat. Res. 730, 59-67 (2012).

926 23. Sanders, J. L. \& Newman, A. B. Telomere length in epidemiology: a biomarker of aging,

927 age-related disease, both, or neither? Epidemiol. Rev. 35, 112-131 (2013).

928 24. Cunningham, J. M. et al. Telomere length varies by DNA extraction method: implications

929 for epidemiologic research. Cancer Epidemiol. Biomarkers Prev. 22, 2047-2054 (2013).

930 25. Blackburn, E. H., Epel, E. S. \& Lin, J. Human telomere biology: A contributory and

931 interactive factor in aging, disease risks, and protection. Science 350, 1193-1198 (2015).

932 26. Gottschling, D. E., Aparicio, O. M., Billington, B. L. \& Zakian, V. A. Position effect at S.

933 cerevisiae telomeres: reversible repression of Pol II transcription. Cell 63, 751-762 (1990).

934 27. Baur, J. A., Zou, Y., Shay, J. W. \& Wright, W. E. Telomere position effect in human cells.

$935 \quad$ Science 292, 2075-2077 (2001).

936 28. Robin, J. D. et al. Telomere position effect: regulation of gene expression with progressive

937 telomere shortening over long distances. Genes Dev. 28, 2464-2476 (2014).

938 29. Buxton, J. L. et al. Human leukocyte telomere length is associated with DNA methylation

939 levels in multiple subtelomeric and imprinted loci. Sci. Rep. 4, 4954 (2014).

940 30. Flores, I., Benetti, R. \& Blasco, M. A. Telomerase regulation and stem cell behaviour. Curr.

941 Opin. Cell Biol. 18, 254-260 (2006).

942 31. Aeby, E., Ahmed, W., Redon, S., Simanis, V. \& Lingner, J. Peroxiredoxin 1 Protects

943 Telomeres from Oxidative Damage and Preserves Telomeric DNA for Extension by

944 Telomerase. Cell Rep. 17, 3107-3114 (2016).

945 32. Tigchelaar, E. F. et al. Cohort profile: LifeLines DEEP, a prospective, general population 946 cohort study in the northern Netherlands: study design and baseline characteristics. BMJ 
Open 5, e006772 (2015).

948 33. Baerlocher, G. M., Vulto, I., de Jong, G. \& Lansdorp, P. M. Flow cytometry and FISH to 949 measure the average length of telomeres (flow FISH). Nat. Protoc. 1, 2365-2376 (2006).

950 34. Araki, T. et al. Normal thymus in adults: appearance on CT and associations with age, sex, 951 BMI and smoking. Eur. Radiol. 26, 15-24 (2016).

952 35. Hannum, G. et al. Genome-wide methylation profiles reveal quantitative views of human 953 aging rates. Mol. Cell 49, 359-367 (2013).

954 36. Ou, X.-L. et al. Predicting human age with bloodstains by sjTREC quantification. PLoS One $955 \quad$ 7, e42412 (2012).

956 37. Bischoff, C. et al. The heritability of telomere length among the elderly and oldest-old. Twin 957 Res. Hum. Genet. 8, 433-439 (2005).

958 38. Andrew, T. et al. Mapping genetic loci that determine leukocyte telomere length in a large 959 sample of unselected female sibling pairs. Am. J. Hum. Genet. 78, 480-486 (2006).

960 39. Lee, J. H. et al. Genome wide association and linkage analyses identified three loci-4q25, 961 17q23.2, and 10q11.21 — associated with variation in leukocyte telomere length: the Long 962 Life Family Study. Frontiers in Genetics vol. 4 (2014).

963 40. Yang, J., Lee, S. H., Goddard, M. E. \& Visscher, P. M. GCTA: a tool for genome-wide 964 complex trait analysis. Am. J. Hum. Genet. 88, 76-82 (2011).

965 41. Scholtens, S. et al. Cohort Profile: LifeLines, a three-generation cohort study and biobank. 966 Int. J. Epidemiol. 44, 1172-1180 (2015).

967 42. Mitchell, C., Schneper, L. M. \& Notterman, D. A. DNA methylation, early life environment, 968 and health outcomes. Pediatr. Res. 79, 212-219 (2016).

969 43. Joubert, B. R. et al. DNA Methylation in Newborns and Maternal Smoking in Pregnancy: 
970 Genome-wide Consortium Meta-analysis. Am. J. Hum. Genet. 98, 680-696 (2016).

971 44. Markunas, C. A. et al. Maternal Age at Delivery Is Associated with an Epigenetic Signature

972 in Both Newborns and Adults. PLoS One 11, e0156361 (2016).

973 45. Joubert, B. R. et al. Maternal smoking and DNA methylation in newborns: in utero effect or 974 epigenetic inheritance? Cancer Epidemiol. Biomarkers Prev. 23, 1007-1017 (2014).

975 46. Wiklund, P. et al. DNA methylation links prenatal smoking exposure to later life health 976 outcomes in offspring. Clin. Epigenetics 11, 97 (2019).

977 47. Xie, K. et al. Epigenetic alterations in longevity regulators, reduced life span, and 978 exacerbated aging-related pathology in old father offspring mice. Proc. Natl. Acad. Sci. U. 979 S. A. 115, E2348-E2357 (2018).

980 48. Moore, A. M. et al. Persistent epigenetic changes in adult daughters of older mothers.

$981 \quad$ Epigenetics 14, 467-476 (2019).

982 49. Oelen, R. et al. Single-cell RNA-sequencing reveals widespread personalized, context-

983 specific gene expression regulation in immune cells. bioRxiv (2021)

984 doi:10.1101/2021.06.04.447088.

985 50. Hao, Y. et al. Integrated analysis of multimodal single-cell data. Cell 184, 3573-3587.e29

$986 \quad$ (2021).

987 51. Nittis, T. et al. Revealing novel telomere proteins using in vivo cross-linking, tandem

988 affinity purification, and label-free quantitative LC-FTICR-MS. Mol. Cell. Proteomics 9 , $989 \quad 1144-1156(2010)$.

990 52. Sekiya, T. et al. The nuclear orphan receptor Nr4a2 induces Foxp3 and regulates 991 differentiation of CD4+ T cells. Nat. Commun. 2, 269 (2011).

992 53. Pellegrino-Coppola, D. et al. Correction for both common and rare cell types in blood is 
important to identify genes that correlate with age. BMC Genomics 22, 184 (2021).

994 54. Tacutu, R. et al. Human Ageing Genomic Resources: new and updated databases. Nucleic

995 Acids Res. 46, D1083-D1090 (2018).

996 55. Passtoors, W. M. et al. IL7R gene expression network associates with human healthy

997 ageing. Immun. Ageing 12, 21 (2015).

998 56. Nguyen, V., Mendelsohn, A. \& Larrick, J. W. Interleukin-7 and immunosenescence. J. Immunol. Res. 2017, 1-17 (2017).

1000 57. Campaner, S. et al. Cdk2 suppresses cellular senescence induced by the c-myc oncogene. Nat. Cell Biol. 12, 54-9; sup pp 1-14 (2010).

1002 58. Ko, A. et al. Oncogene-induced senescence mediated by c-Myc requires USP10 dependent 1003 deubiquitination and stabilization of p14ARF. Cell Death Differ. 25, 1050-1062 (2018).

1004 59. Shepherd, J. C., Walldorf, U., Hug, P. \& Gehring, W. J. Fruit flies with additional 1005 expression of the elongation factor EF-1 alpha live longer. Proc. Natl. Acad. Sci. U. S. A. 86, 1006 7520-7521 (1989).

1007 60. Vera, M. et al. The translation elongation factor eEF1A1 couples transcription to translation 1008 during heat shock response. Elife 3, e03164 (2014).

1009 61. Begel, O., Boulay, J., Albert, B., Dufour, E. \& Sainsard-Chanet, A. Mitochondrial group II 1010 introns, cytochrome c oxidase, and senescence in Podospora anserina. Mol. Cell. Biol. 19, 1011 4093-4100 (1999).

1012 62. Soro-Arnaiz, I. et al. Role of mitochondrial complex IV in age-dependent obesity. Cell Rep. $1013 \quad \mathbf{1 6}, 2991-3002$ (2016).

1014 63. McHugh, D. \& Gil, J. Senescence and aging: Causes, consequences, and therapeutic 1015 avenues. J. Cell Biol. 217, 65-77 (2018). 
1016

1017

1018

1019

1020

1021

1022

1023

1024

1025

1026

1027

1028

1029

1030

1031

1032

1033

1034

1035

1036

1037

1038

64. Fasching, C. L. Telomere length measurement as a clinical biomarker of aging and disease. Crit. Rev. Clin. Lab. Sci. 55, 443-465 (2018).

65. Yamada, O. \& Kawauchi, K. The role of the JAK-STAT pathway and related signal cascades in telomerase activation during the development of hematologic malignancies. JAKSTAT 2, e25256 (2013).

66. Mazzucchelli, R. \& Durum, S. K. Interleukin-7 receptor expression: intelligent design. Nat. Rev. Immunol. 7, 144-154 (2007).

67. Lodewijckx, I. \& Cools, J. Deregulation of the interleukin-7 signaling pathway in lymphoid malignancies. Pharmaceuticals (Basel) 14, 443 (2021).

68. Gatbonton, T. et al. Telomere length as a quantitative trait: genome-wide survey and genetic mapping of telomere length-control genes in yeast. PLoS Genet. 2, e35 (2006).

69. Askree, S. H. et al. A genome-wide screen for Saccharomyces cerevisiae deletion mutants that affect telomere length. Proc. Natl. Acad. Sci. U. S. A. 101, 8658-8663 (2004).

70. Ungar, L. et al. A genome-wide screen for essential yeast genes that affect telomere length maintenance. Nucleic Acids Res. 37, 3840-3849 (2009).

71. Liu, N.-N., Han, T. X., Du, L.-L. \& Zhou, J.-Q. A genome-wide screen for Schizosaccharomyces pombe deletion mutants that affect telomere length. Cell Res. 20, 963-965 (2010).

72. Abdulkina, L. R. et al. Components of the ribosome biogenesis pathway underlie establishment of telomere length set point in Arabidopsis. Nat. Commun. 10, 5479 (2019).

73. Lessard, F. et al. Senescence-associated ribosome biogenesis defects contributes to cell cycle arrest through the Rb pathway. Nat. Cell Biol. 20, 789-799 (2018).

74. Wild, T. et al. A protein inventory of human ribosome biogenesis reveals an essential 
function of exportin 5 in 60S subunit export. PLoS Biol. 8, e1000522 (2010).

1040

1041

1042

1043

1044

1045

1046

1047

1048

1049

1050

1051

1052

1053

1054

1055

1056

1057

1058

1059

1060

1061

75. Behm-Ansmant, I., Gatfield, D., Rehwinkel, J., Hilgers, V. \& Izaurralde, E. A conserved role for cytoplasmic poly(A)-binding protein 1 (PABPC1) in nonsense-mediated mRNA decay. EMBO J. 26, 1591-1601 (2007).

76. Lew, J. E., Enomoto, S. \& Berman, J. Telomere length regulation and telomeric chromatin require the nonsense-mediated mRNA decay pathway. Mol. Cell. Biol. 18, 6121-6130 (2020).

77. Moyzis, R. K. et al. A highly conserved repetitive DNA sequence, (TTAGGG)n, present at the telomeres of human chromosomes. Proc. Natl. Acad. Sci. U. S. A. 85, 6622-6626 (1988).

78. Cawthon, R. M. Telomere measurement by quantitative PCR. Nucleic Acids Res. 30, e47 (2002).

79. Cawthon, R. M. Telomere length measurement by a novel monochrome multiplex quantitative PCR method. Nucleic Acids Res. 37, e21 (2009).

80. Alder, J. K. et al. Diagnostic utility of telomere length testing in a hospital-based setting. Proc. Natl. Acad. Sci. U. S. A. 115, E2358-E2365 (2018).

81. Daniali, L. et al. Telomeres shorten at equivalent rates in somatic tissues of adults. Nat. Commun. 4, 1597 (2013).

82. Slagboom, P. E., Droog, S. \& Boomsma, D. I. Genetic determination of telomere size in humans: a twin study of three age groups. Am. J. Hum. Genet. 55, 876-882 (1994).

83. Faul, J. D., Mitchell, C. M., Smith, J. A. \& Zhao, W. Estimating Telomere Length Heritability in an Unrelated Sample of Adults: Is Heritability of Telomere Length Modified by Life Course Socioeconomic Status? Biodemography Soc. Biol. 62, 73-86 (2016).

84. Coutts, F. et al. The polygenic nature of telomere length and the anti-ageing properties of 
lithium. Neuropsychopharmacology 44, 757-765 (2019).

1063 85. Njajou, O. T. et al. Telomere length is paternally inherited and is associated with parental

1064 lifespan. Proc. Natl. Acad. Sci. U. S. A. 104, 12135-12139 (2007).

1065 86. Nawrot, T. S., Staessen, J. A., Gardner, J. P. \& Aviv, A. Telomere length and possible link to X chromosome. Lancet $363,507-510$ (2004).

1067 87. Vasa-Nicotera, M. et al. Mapping of a major locus that determines telomere length in humans. Am. J. Hum. Genet. 76, 147-151 (2005).

1069 88. Aston, K. I. et al. Divergence of sperm and leukocyte age-dependent telomere dynamics: implications for male-driven evolution of telomere length in humans. Mol. Hum. Reprod. 18, 517-522 (2012).

1072 89. Kimura, M. et al. Offspring's leukocyte telomere length, paternal age, and telomere 1073 elongation in sperm. PLoS Genet. 4, e37 (2008).

1074 90. Eisenberg, D. T. A., Hayes, M. G. \& Kuzawa, C. W. Delayed paternal age of reproduction 1075 in humans is associated with longer telomeres across two generations of descendants. Proc. 1076 Natl. Acad. Sci. U. S. A. 109, 10251-10256 (2012).

1077 91. Yang, Z. et al. SOX11: friend or foe in tumor prevention and carcinogenesis? Ther. Adv. $1078 \quad$ Med. Oncol. 11, 1758835919853449 (2019).

1079 92. Foronda, M. et al. Sox4 links tumor suppression to accelerated aging in mice by modulating $1080 \quad$ stem cell activation. Cell Rep. 8, 487-500 (2014).

1081 93. Gardner, M. et al. Gender and telomere length: systematic review and meta-analysis. Exp. 1082 Gerontol. 51, 15-27 (2014).

1083 94. Valdes, A. M. et al. Obesity, cigarette smoking, and telomere length in women. Lancet 366, 1084 $662-664$ (2005). 
1085 95. Patel, C. J., Manrai, A. K., Corona, E. \& Kohane, I. S. Systematic correlation of

1086 environmental exposure and physiological and self-reported behaviour factors with

1087 leukocyte telomere length. Int. J. Epidemiol. 46, 44-56 (2017).

1088 96. Bakaysa, S. L. et al. Telomere length predicts survival independent of genetic influences.

$1089 \quad$ Aging Cell 6, 769-774 (2007).

1090 97. Cawthon, R. M., Smith, K. R., O’Brien, E., Sivatchenko, A. \& Kerber, R. A. Association

1091 between telomere length in blood and mortality in people aged 60 years or older. Lancet

$1092 \quad 361,393-395$ (2003).

1093 98. Zhernakova, D. V. et al. Individual variations in cardiovascular-disease-related protein

1094 levels are driven by genetics and gut microbiome. Nat. Genet. 50, 1524-1532 (2018).

1095 99. Das, S. et al. Next-generation genotype imputation service and methods. Nat. Genet. 48,

1096 1284-1287 (2016).

1097 100.Consortium, T. H. R. \& the Haplotype Reference Consortium. A reference panel of 64,976

1098 haplotypes for genotype imputation. Nature Genetics vol. 48 1279-1283 (2016).

1099 101. Bonder, M. J. et al. Disease variants alter transcription factor levels and methylation of their

$1100 \quad$ binding sites. Nat. Genet. 49, 131-138 (2017).

1101 102. Touleimat, N. \& Tost, J. Complete pipeline for Infinium® Human Methylation 450K

1102 BeadChip data processing using subset quantile normalization for accurate DNA

1103 methylation estimation. Epigenomics 4, 325-341 (2012).

1104 103.Fehrmann, R. S. N. et al. Trans-eQTLs reveal that independent genetic variants associated 1105 with a complex phenotype converge on intermediate genes, with a major role for the HLA.

$1106 \quad$ PLoS Genet. 7, e1002197 (2011).

1107 104.Chang, C. C. et al. Second-generation PLINK: rising to the challenge of larger and richer 
1108 datasets. Gigascience 4, 7 (2015).

1109 105.Benjamini, Y. \& Hochberg, Y. Controlling the false discovery rate: a practical and powerful

1110 approach to multiple testing. J. R. Stat. Soc. (1995).

1111 106. Friedman, J., Hastie, T. \& Tibshirani, R. Regularization Paths for Generalized Linear

1112 Models via Coordinate Descent. J. Stat. Softw. 33, 1-22 (2010).

1113 107. Yamamoto, T., Hirose, K., Keele, L. \& Imai, K. Mediation: R package for causal mediation 1114 analysis. of Statistical Software (2014).

1115 108.Zheng, G. X. Y. et al. Massively parallel digital transcriptional profiling of single cells. Nat.

1116 Commun. 8, 14049 (2017).

1117 109. Satija, R., Farrell, J. A., Gennert, D., Schier, A. F. \& Regev, A. Spatial reconstruction of 1118 single-cell gene expression data. Nat. Biotechnol. 33, 495-502 (2015).

1119 110.Finak, G. et al. MAST: a flexible statistical framework for assessing transcriptional changes 1120 and characterizing heterogeneity in single-cell RNA sequencing data. Genome Biol. 16, 278 $1121 \quad$ (2015).

1122 111.Liao, Y., Wang, J., Jaehnig, E. J., Shi, Z. \& Zhang, B. WebGestalt 2019: gene set analysis 1123 toolkit with revamped UIs and APIs. Nucleic Acids Res. 47, W199-W205 (2019).

1124 112.Smedley, D. et al. BioMart--biological queries made easy. BMC Genomics 10, 22 (2009). 


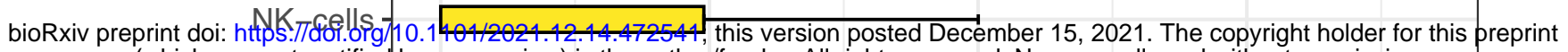

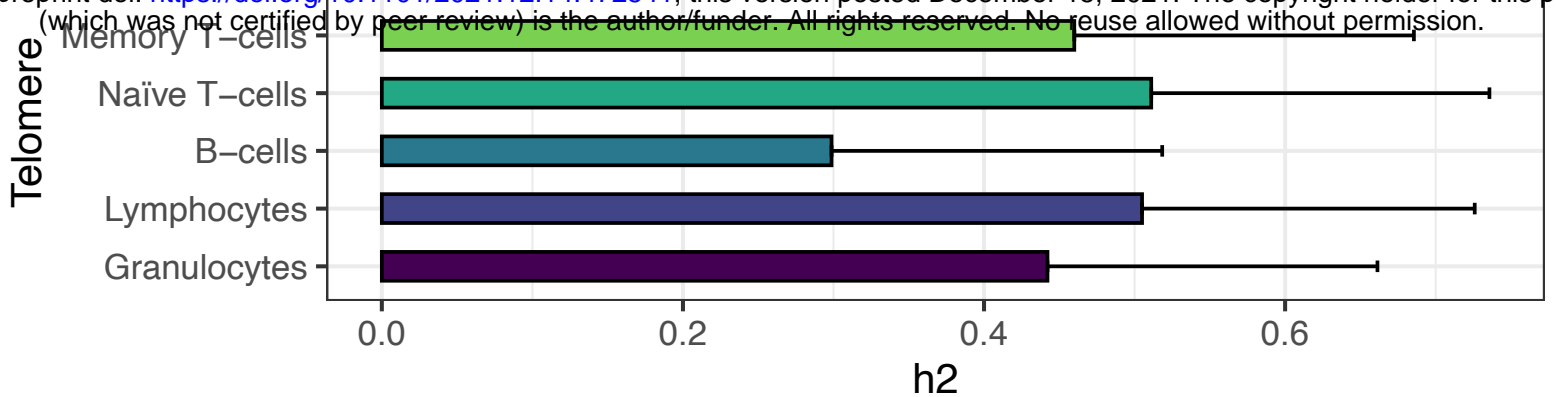

B

rs33961405 - TERT gene
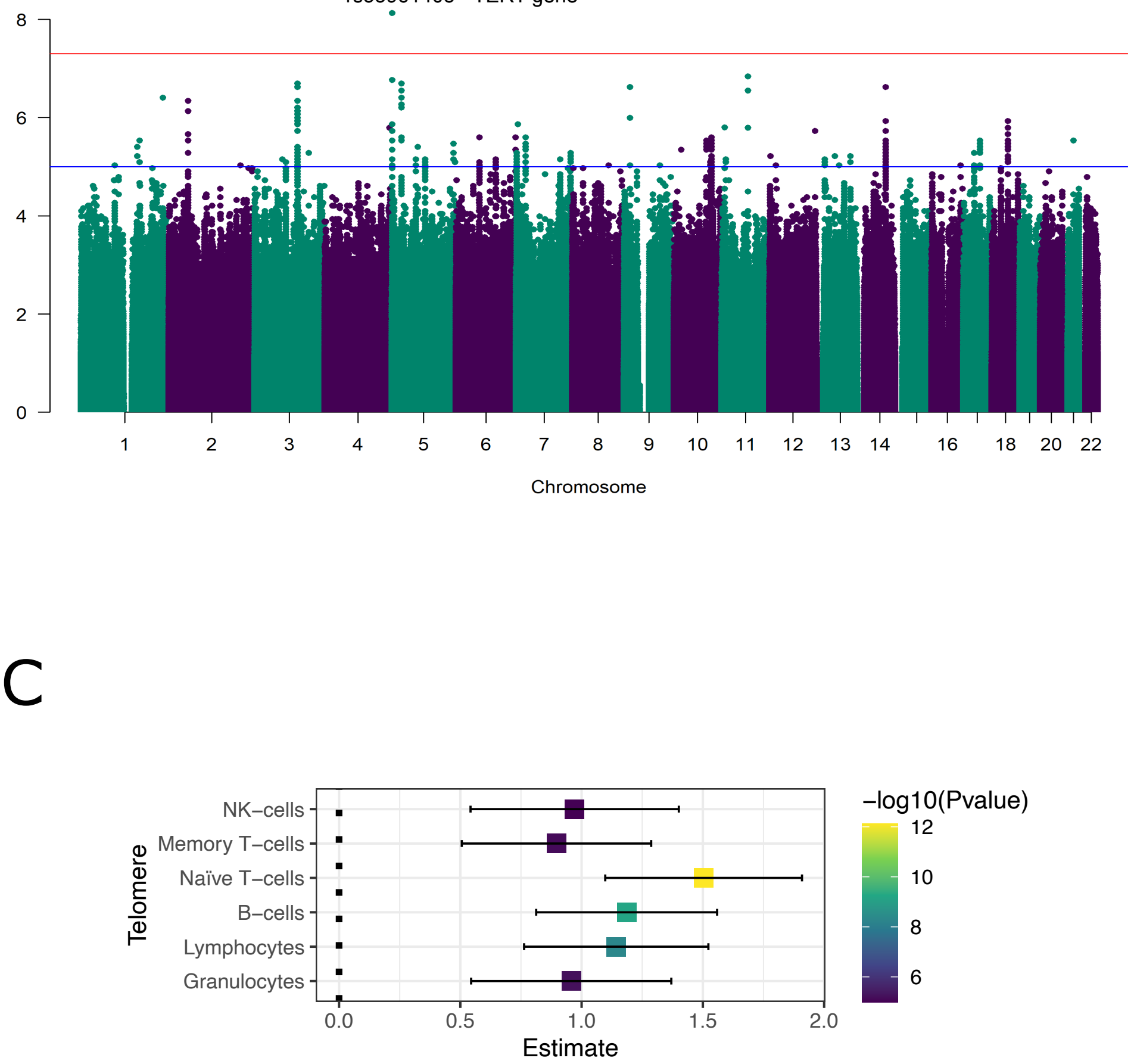


\section{A}

Weight(Kg)

bioRxiv preprint doi: https://doi.orito

0.1101/2021.12.14.472541; thigmosiagenasted December 15, 2021. The copyright holder for this preprint (which was not certified by peer review) is the author/fundef. All rights reserved. No reuse allowed without permission.

Waist circumference $(\mathrm{cm})$

\section{Pulse rate(per m
Poorly healing
wounds in feet(yes}

Poorly healing
wounds in feet(yes/no)

Mother smoke
while pregnant(categories)

Father smoke(yes/no)

Any parent smoke(yes/no)

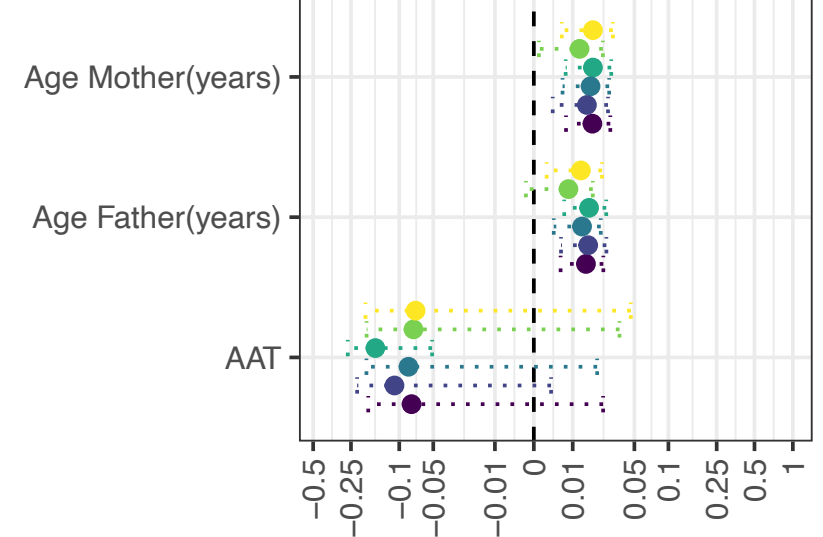

Estimated effect on telomere length
- Memory_T-cells

Naïve_T-cells

NK-cells

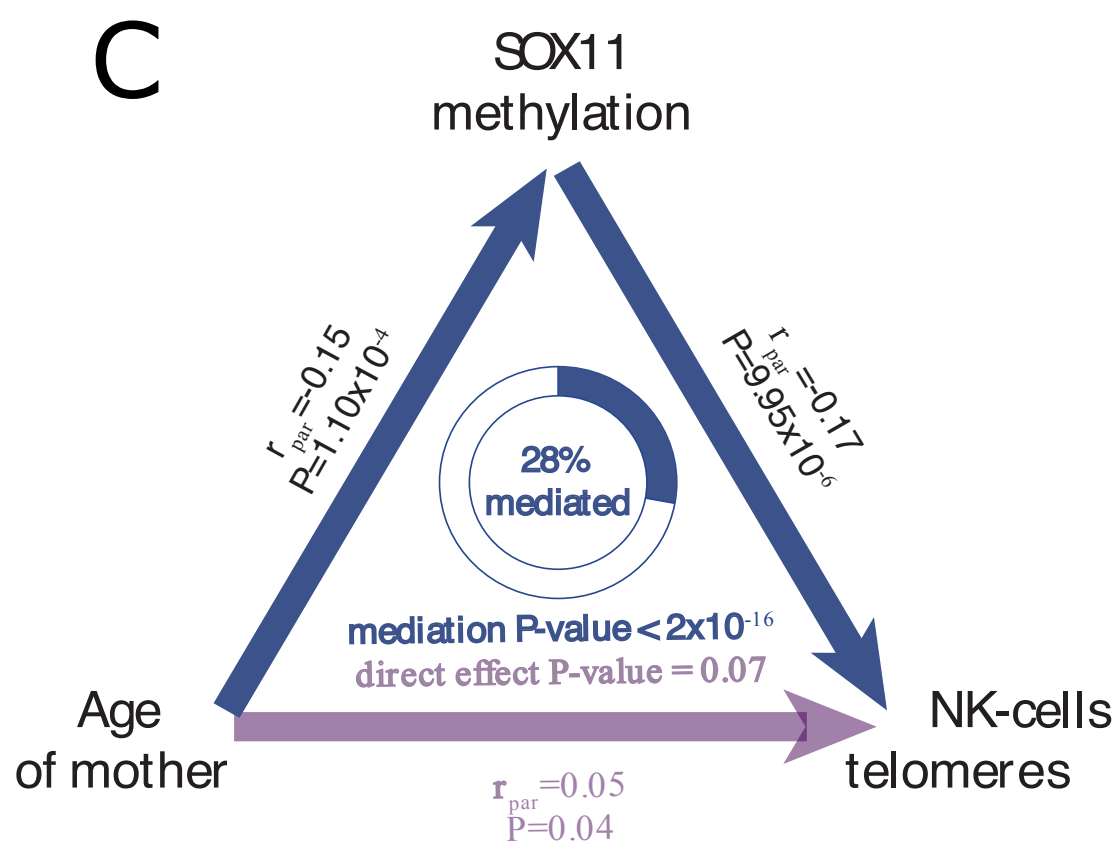

$B$

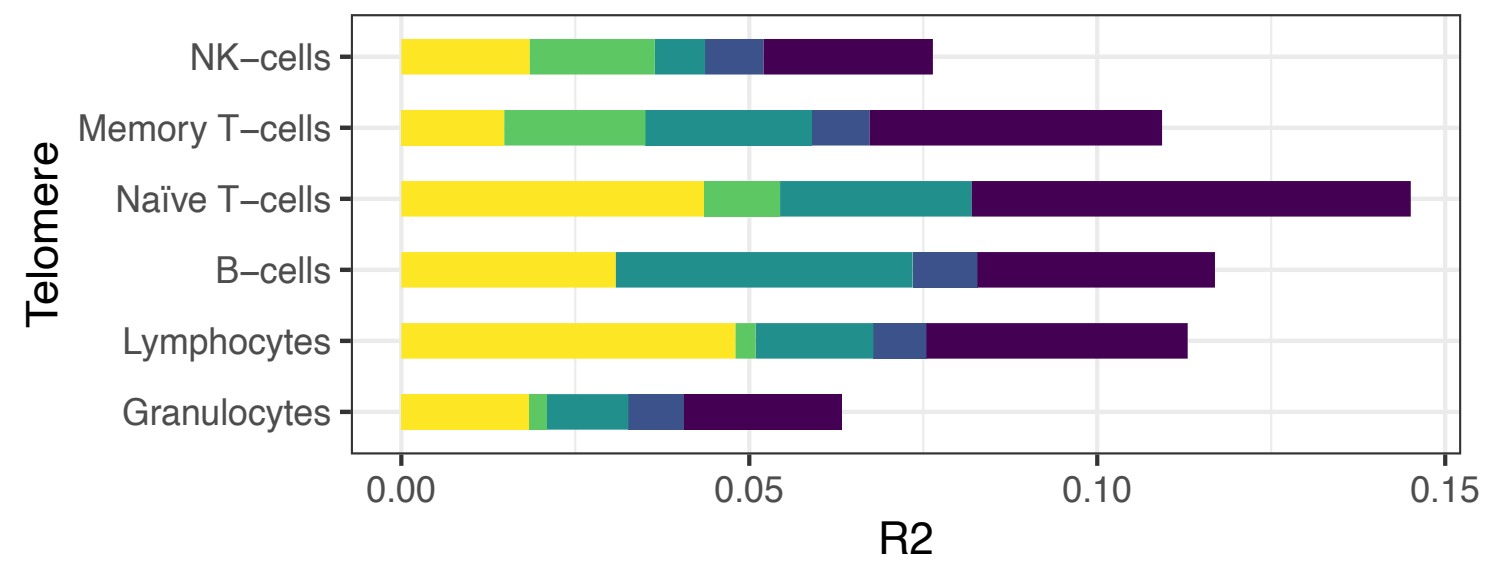

Model

Genetics

Parental

BMI

Cells

Sex 

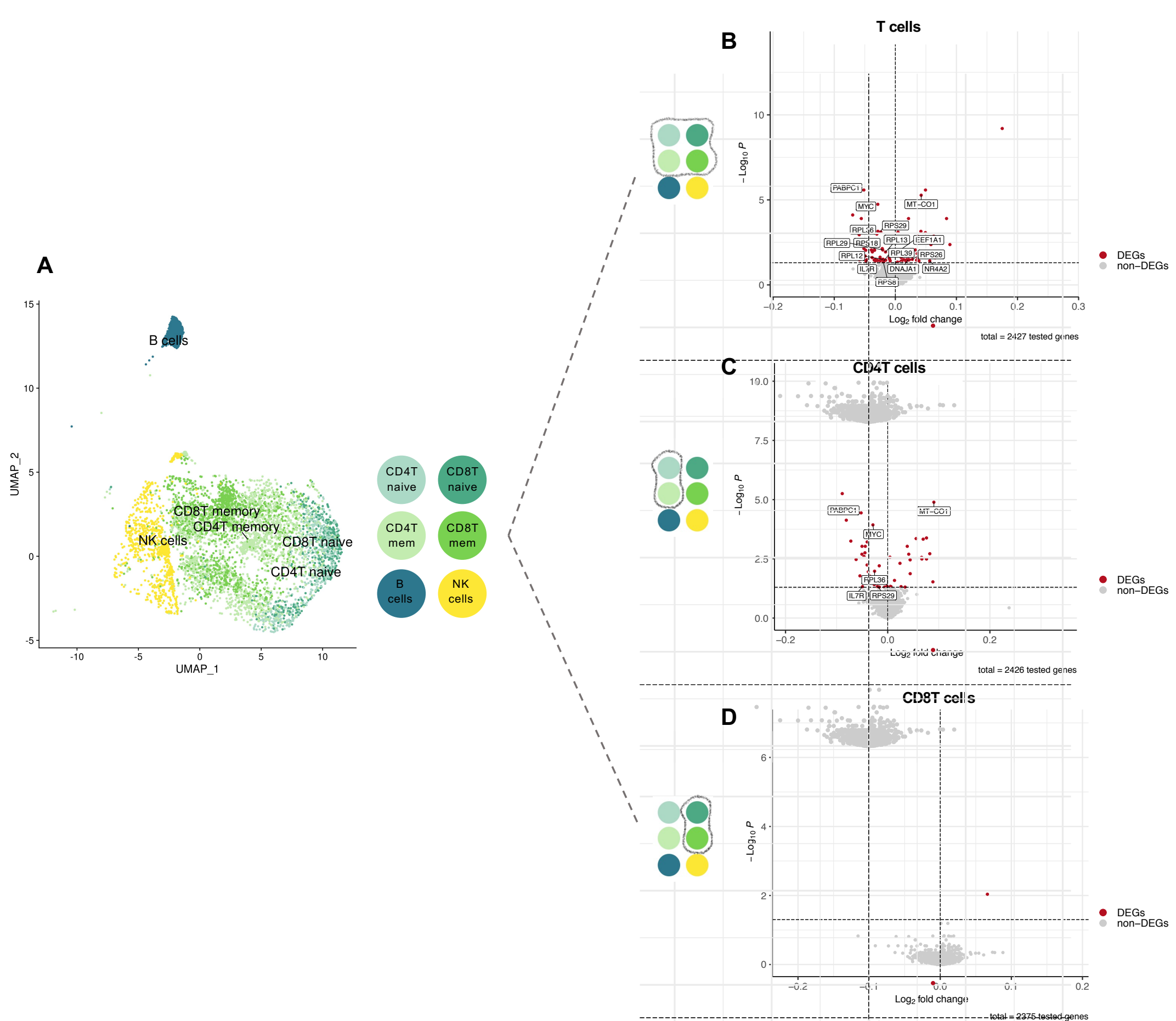

E

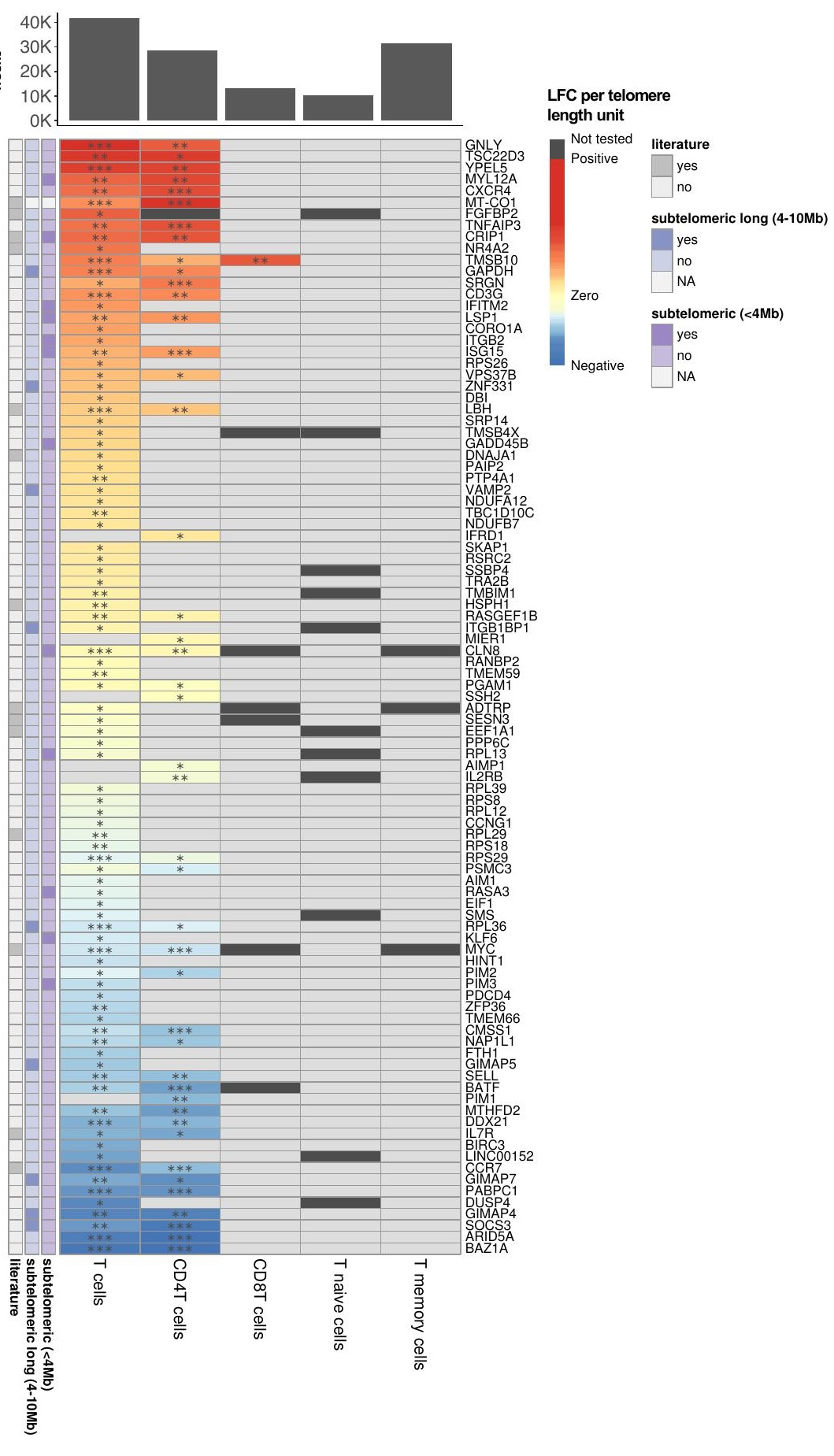


